ACCEPTED TO The Astrophysical Journal

Preprint typeset using $\mathrm{LT}_{\mathrm{E}} \mathrm{X}$ style emulateapj v. 08/13/06

\title{
A RADIO AND OPTICAL POLARIZATION STUDY OF THE MAGNETIC FIELD IN THE SMALL MAGELLANIC
} CLOUD

\author{
S. A. Mao, ${ }^{1}$ B. M. Gaensler,${ }^{2,8}$ S. Stanimirović,${ }^{3}$ M. HaVerkorn,${ }^{4,9}$ N. M. MCClure-Griffiths,${ }^{5}$ L. Staveley-Smith ${ }^{6,10}$ And \\ J. M. DICKEY ${ }^{7}$ \\ Accepted to The Astrophysical Journal
}

\begin{abstract}
We present a study of the magnetic field of the Small Magellanic Cloud (SMC), carried out using radio Faraday rotation and optical starlight polarization data. Consistent negative rotation measures (RMs) across the SMC indicate that the line-of-sight magnetic field is directed uniformly away from us with a strength $0.19 \pm 0.06 \mu \mathrm{G}$. Applying the Chandrasekhar-Fermi method to starlight polarization data yields an ordered magnetic field in the plane of the sky of strength $1.6 \pm 0.4 \mu \mathrm{G}$ oriented at a position angle $4^{\circ} \pm 12^{\circ}$, measured counter-clockwise from the great circle on the sky joining the SMC to the Large Magellanic Cloud (LMC). We construct a three-dimensional magnetic field model of the SMC, under the assumption that the RMs and starlight polarization probe the same underlying large-scale field. The vector defining the overall orientation of the SMC magnetic field shows a potential alignment with the vector joining the center of the SMC to the center of the LMC, suggesting the possibility of a "pan-Magellanic" magnetic field. A cosmic-ray driven dynamo is the most viable explanation of the observed field geometry, but has difficulties accounting for the observed uni-directional field lines. A study of Faraday rotation through the Magellanic Bridge is needed to further test the pan-Magellanic field hypothesis.
\end{abstract}

Subject headings: magnetic fields —Faraday rotation—polarization—galaxies: Small Magellanic Cloud

\section{INTRODUCTION}

Magnetic fields play key roles in many astrophysical processes in the interstellar medium (ISM) - they accelerate and confine cosmic rays, trigger star formation and exert pressure to balance against gravity (Beck 2007). Therefore, to better understand galaxy evolution, investigating the structure, origin and evolution of galactic magnetic fields is necessary.

It is useful to picture the total magnetic field at any location in a galaxy as a superposition of an ordered large-scale component and a random small-scale component. An ordered (or uniform) field can either be coherent or incoherent: a coherent field has unidirectional field lines, whereas an incoherent field has field lines of the same orientation but has frequent field reversals. A large-scale dynamo is the only known mechanism that can generate large-scale coherent fields (Beck 2004).

Coherent magnetic fields have been observed in normal spiral galaxies such as the Milky Way and M31. The fields are typically in spiral-like configurations with field strengths of a few $\mu \mathrm{G}$ (Beck 2007). Because these galaxies have significant differential rotation, such observations can be explained by the standard $\alpha-\omega$ dynamo which amplifies and orders the field by small scale turbulent motion (the $\alpha$-effect) as well as differential rotation (the $\omega$-effect) in the galactic disk on a

\footnotetext{
${ }^{1}$ Harvard-Smithsonian Center for Astrophysics, Cambridge, MA 02138 samao@cfa.harvard.edu

${ }^{2}$ School of Physics, The University of Sydney, NSW 2006, Australia

${ }^{3}$ Department of Astronomy, University of Wisconsin, Madison, WI 53706

${ }^{4}$ Astronomy Department, University of California, Berkeley, CA 94720

${ }^{5}$ Australia Telescope National Facility, CSIRO, Epping, NSW 1710, Australia

${ }^{6}$ School of Physics, University of Western Australia, Crawley, WA 6009, Australia

${ }^{7}$ Physics Department, University of Tasmania, Hobart, TAS 7001, Australia

${ }^{8}$ Alfred P. Sloan Research Fellow, Australian Research Council Federation Fellow

9 Jansky Fellow, National Radio Astronomy Observatory

${ }^{10}$ Premier's Fellow
}

global e-folding time of $\sim 10^{9}$ yrs (Shukurov 2007). Despite its success in accounting for the large scale coherent field seen in spiral galaxies, the standard dynamo theory fails to explain the presence of coherent magnetic fields discovered in several irregular galaxies such as NGC 4449 and the Large Magellanic Cloud (LMC), due to its long amplification time scale (Klein et al. 1996; Chyży et al. 2000; Gaensler et al. 2005).

The polarized radio continuum emission of NGC 4449, a dwarf irregular galaxy, at 4.9 and $8.6 \mathrm{GHz}$ reveals large scale spiral like structure in the magnetic field. Moreover, this slowly rotating galaxy shows regions of coherent magnetic field from Faraday rotation studies (Klein et al. 1996). The long field amplification time scale of the classical mean field dynamo argues against this being the underlying mechanism that produces the magnetic field observed in NGC 4449. A Faraday rotation measure study of extragalactic polarized sources behind the LMC carried out by Gaensler et al. (2005) suggests that the LMC hosts a coherent axisymmetric magnetic field of strength $\sim 1 \mu \mathrm{G}$. The random component dominates over the ordered component with a strength of $\sim 3 \mu \mathrm{G}$. It is believed that close encounters between the Magellanic Clouds and the Milky Way have triggered episodes of star formation in the LMC over the past 4 billion years (Bekki $\&$ Chiba 2005). Any coherent field built up by the standard dynamo would have been disrupted by the outflow from active star forming regions. Hence, the existence of a coherent field in the LMC suggests that a field generation mechanism with a much faster amplification time scale is at work.

A much more efficient process, the Parker (1992) dynamo, could account for the large-scale magnetic fields detected in irregular galaxies such as NGC 4449 and the LMC (Hanasz et al. 2004). Vertical pressure from cosmic rays can force magnetic field lines into the galactic halo and form loops which reconnect and then are amplified by the $\omega$ effect. This process can significantly increase the $\alpha$ effect and can operate over a much shorter amplification time scale than the $\alpha-\omega$ dynamo (Hanasz et al. 2004). 
As a close neighbor of the Milky Way, the large angular extent of the Small Magellanic Cloud (SMC) on the sky allows us to determine RMs of polarized background radio sources whose projections lie behind it. Since RM is an integral of the line of sight magnetic field strength weighted by the thermal electron density, only a coherent field can produce a consistent sign in RM as a function of position on the sky. An RM study can distinguish between coherent and incoherent fields and can indicate the geometry of any coherent field, and therefore can potentially reveal the field generation mechanism.

The alignment of non-spherical dust grains with the magnetic field in the ISM linearly polarizes optical radiation that travels through it. Therefore, measuring the optical polarization of stars in the SMC enables us to estimate the orientation of the ordered magnetic field in the plane of the sky. The spread in polarization position angle of an ensemble of starlight polarization measurements allows one to estimate the mean strength of the ordered component of the magnetic field using the Chandrasekhar-Fermi (1953), or C-F method. Assuming that the field is unidirectional and knowing both the line-of-sight and the plane-of-the-sky magnetic field strength and orientation, one can construct a three dimensional magnetic field vector for the SMC, which can further constrain the field generation mechanism.

In this paper, we present the results of a radio and optical polarization study of the magnetic field in the SMC. We use RMs of polarized extragalactic background radio sources to determine the magnetic field strength and direction along the line of sight. The orientation and strength of the plane-of-thesky magnetic field is studied using optical polarization of stars in the SMC. We start in $\S 1.1$ by reviewing the properties of the SMC and we summarize previous studies on SMC's magnetism in $\S 1.2$. In $\S 1.3$ and $\S 1.4$, we summarize the physics behind the RM method and the C-F method respectively. We then describe the observation, data reduction procedures and present results in $\S 2$. We derive the line-of-sight magnetic field of the SMC in $\S 3$. In $\S 4$, we estimate the plane-of-thesky magnetic field and the random field strength in the SMC. A 3D magnetic field vector of the SMC is constructed in $\S 5$. A discussion of possible field generation mechanisms is provided in $\S 6$.

Throughout this paper, we represent physical quantities in the plane of the sky by the subscript $\perp$ and those along the line of sight by the subscript $\|$. We denote the average of a quantity $x$ over the plane of the sky and that averaged along the line of sight by $\langle x\rangle$ and $\bar{x}$ respectively. Table 1 contains a glossary of the variables used in this paper.

\subsection{The Small Magellanic Cloud}

The Small Magellanic Cloud is a nearby gas rich dwarf irregular galaxy. Recent precise measurements of apparent magnitudes of stars at the tip of the red giant branch in the SMC yield a distance modulus of 18.99 \pm 0.03 (formal) \pm 0.08 (systematic) (Cioni et al. 2000), which corresponds to a distance of roughly $63 \pm 1 \mathrm{kpc}$. In this paper, we adopt a distance to the SMC of $60 \mathrm{kpc}$. Basic parameters of the SMC are listed in Table 2. Both the SMC and the LMC are thought to be satellite galaxies of the Milky Way. However, a recent study by Besla et al. (2007) suggests that the Clouds are not bound to the Milky Way but are on their first passage about the Galaxy. It is still of great debate as to whether the Magellanic Clouds formed as a binary, or whether they became dynamically coupled to each other $\sim 4$ Gyrs ago (Bekki $\&$ Chiba 2005). The most recent proper motion measurements of the Clouds suggest that both scenarios are equally probable (Kallivayalil et al. 2006; Piatek et al. 2008). It is believed that the last close encounter of the Magellanic Clouds $\sim 0.2$ Gyrs ago triggered star formation in the SMC and created the morphological and kinematic features seen in the present day SMC (Yoshizawa \& Noguchi 2003).

Stanimirović et al. (2004) found that the depth of the SMC is within its tidal radius ( $\sim 4-9 \mathrm{kpc})$. Lah et al. (2005) have measured distances to pulsating red giants in the SMC and found a distance scatter of $3.2 \pm 1.6 \mathrm{kpc}$, which agrees with the results of Stanimirović et al. (2004). N-body simulations of the gravitational interaction between the LMC, SMC and the Milky Way have been able to reproduce the large line-ofsight extent of the SMC and its two tidal arms (Gardiner et al. 1994; Yoshizawa \& Noguchi 2003).

The gas component in the SMC shows signs of rotation whereas the old stellar component does not (Hatzidimitriou et al. 1997). The gas kinematics of the SMC were investigated by Stanimirović et al. (2004), who found a strong velocity gradient in $\mathrm{HI}$ across the SMC from the southwest to the northeast. Stanimirović et al. (2004) constructed a rotation curve of the gas disk, and derived a maximum rotation velocity of $50 \mathrm{~km} \mathrm{~s}^{-1}$.

\subsection{Previous Studies of Magnetism in the $S M C$}

The most common way to study magnetic fields in external galaxies is by observing synchrotron emission at radio wavelengths. Haynes et al. (1986) examined linear polarization maps of the SMC at $1.4 \mathrm{GHz}$. They found, without any Faraday rotation correction, an ordered magnetic field directed along the SMC's bar in the plane of the sky. Loiseau et al. (1987) analyzed radio continuum maps of the SMC at 408 $\mathrm{MHz}, 1.4 \mathrm{GHz}$ and $2.3 \mathrm{GHz}$ and obtained a total equipartition field strength of $\sim 5 \mu \mathrm{G}$ by using an average non-thermal spectral index $\alpha$ of 0.87 (specific intensity of synchrotron emission $\left.I_{\nu} \propto \nu^{-\alpha}\right)$ and a depth of the synchrotron emitting region of $6 \mathrm{kpc}$. Haynes et al. (1990) observed the SMC at $2.5,4.8$ and $8.6 \mathrm{GHz}$ and concluded that the SMC has a large-scale magnetic field, since weak polarized emission is detected across the whole SMC body.

Chi \& Wolfendale (1993) measured the $\gamma$-ray flux from the SMC to determine the field strength from radio synchrotron emission without needing to invoke the equipartition assumption. They obtained an estimate which exceeded the equipartition value and concluded that energy equipartition is not valid in the SMC. Pohl (1993), however, took the energy density in cosmic ray electrons into account, and demonstrated that energy equipartition between magnetic field and cosmic rays is not necessarily violated.

One should note that calculating the total equipartition field requires knowledge of the depth of the synchrotron emitting layer of the SMC and the inclination of magnetic field with respect to the plane of the sky, which are both poorly constrained in the SMC. Also, as explained by Beck \& Krause (2005), the classical equipartition energy formula underestimates the true equipartition field strength, since the former involves integrating the radio spectrum with a fixed frequency interval instead of a fixed energy interval, and with insufficient knowledge of the ratio of the total energy density of cosmic ray nuclei to that of the electrons and positrons. We will further explore this issue in $\S 4.2$.

Optical polarization from stars in the SMC can be used to map the geometry of the plane-of-the-sky component of the magnetic field, assuming that the observed polarization 
is due to scattering by non-spherical foreground dust grains aligned by the local magnetic field. Polarization measurements for 147 SMC stars have been made by Mathewson \& Ford (1970a,b), Schmidt (1970, 1976), and Magalhães et al. (1990). Since the polarization "vectors" ${ }^{1}$, after removal of Galactic foreground polarization, appear to run parallel to the direction connecting the Magellanic Clouds (Figure 1), the "Pan-Magellanic" magnetic field hypothesis emerged, which suggests the existence of a large scale magnetic field associated with the entire Magellanic system. Wayte 's (1990) reanalysis of previously obtained starlight polarization data sets appeared to support the idea of this Pan-Magellanic magnetic field. However, as the Galactic foreground polarization also runs along the projection of the line joining the Magellanic Clouds (Schmidt 1970), any contribution from Galactic foreground that has not been correctly subtracted could be misinterpreted as an intrinsic magnetic field connecting the Magellanic Clouds. In addition, anisotropic scattering in the ISM may also polarize starlight (Widrow 2002). Therefore, one has to be cautious interpreting these results. In $\S 4.1$, we will further analyze the optical starlight polarization data using the $\mathrm{C}-\mathrm{F}$ method to derive the ordered magnetic field strength of the SMC in the plane of the sky.

\subsection{Faraday Rotation}

When linearly polarized light travels through a magnetized plasma, the plane of polarization rotates due to birefringence. The change in the polarization position angle $\Delta \phi$ in radians is given by

$$
\Delta \phi=\mathrm{RM} \lambda^{2}
$$

where $\lambda$ is the wavelength of the radiation measured in meters and $\mathrm{RM}$ is the rotation measure, defined by

$$
\mathrm{RM}=0.812 \int_{\text {source }}^{\text {observer }} n_{e}(l) B_{\|}(l) d l \mathrm{rad} \mathrm{m}^{-2}
$$

In the above equation, $n_{e}(l)\left(\right.$ in $\left.\mathrm{cm}^{-3}\right)$ is the thermal electron density, $B_{\|}(l)$ (in $\mu \mathrm{G}$ ) is the line of sight magnetic field strength and $\mathrm{d} l$ (in pc) is a line element along the line of sight. The sign of the RM gives the direction of the line of sight component of the average field. For example, a negative RM represents a field whose line of sight component is directed away from us.

RMs for extragalactic radio sources behind the SMC can be decomposed into various contributions along the line of sight: the intrinsic RM of the source, the RM through the intergalactic medium (IGM), the RM through the SMC, and the foreground Milky Way RM.

$$
\mathrm{RM}_{\text {observed }}=\mathrm{RM}_{\text {Intrinsic }}+\mathrm{RM}_{\mathrm{IGM}}+\mathrm{RM}_{\mathrm{SMC}}+\mathrm{RM}_{\text {MilkyWay }}
$$

$\mathrm{RM}_{\text {MilkyWay }}$ can be estimated by observing RMs of extragalactic sources whose projections on the sky lie outside, but close to the SMC. RMs of extragalactic sources at Galactic latitudes $|b|>30^{\circ}$ have a standard deviation $\sim 10 \mathrm{rad} \mathrm{m}^{-2}$ (Johnston-Hollitt et al. 2004). In addition, Broten et al. (1988) showed that the extragalactic RMs in the neighborhood of the SMC have $\left|R M_{\text {Intrinsic }}+\mathrm{RM}_{\mathrm{IGM}}+\mathrm{RM}_{\text {MilkyWay }}\right| \leq 25 \mathrm{rad} \mathrm{m}^{-2}$. This implies that the intrinsic RM and the RM through the IGM are both small compared to the statistical errors of our RM measurements (See Table 3). After the removal of the

\footnotetext{
${ }^{1}$ Position angles provide information on the orientation of the polarization plane, but not the direction. Hence, there is a $180^{\circ}$ direction ambiguity.
}

Galactic foreground, the observed RM should adequately represent the RM through the SMC. Ionospheric Faraday rotation may also contaminate our data. However, since the magnitude of RM induced by the ionosphere is typically only $\sim 1$ rad $\mathrm{m}^{-2}$ (Tinbergen 1996), this is not of great concern in our experiment as the statistical errors of our RM measurements greatly exceed this value (See Table 3 ).

Faraday rotation is complementary to other measurement techniques such as equipartition, synchrotron intensity and starlight polarization since RMs provide the direction of the magnetic field (and hence the field coherency), while other techniques only provide the field orientation and estimates of the field strength, but not its direction. With independent knowledge of the thermal electron density and the line of sight depth of the SMC, one can estimate the average line-of-sight magnetic field strength using Equation (2) assuming that there is no correlation between electron density and magnetic field on small scales. If such correlation or anti-correlation exists, it will result in either underestimation or overestimation of the field strength by a factor of up to two to three (Beck et al. 2003).

\subsection{Optical Starlight Polarization and the Chandrasekhar-Fermi Method}

Starlight polarization alone does not directly give the magnetic field strength. However, measuring the spread in polarization position angles for an ensemble of stars allows one to estimate the mean strength of the ordered component of the magnetic field (Chandrasekhar \& Fermi 1953). This technique assumes that the magnetic field is frozen into the gas and that turbulence leads to isotropic fluctuation of the magnetic field around the mean field direction (Heitsch et al. 2001; Sandstrom 2001). Assuming equipartition between the turbulent kinetic and the magnetic energy, the ordered magnetic field strength averaged over the plane of the sky, $\left\langle B_{o, \perp}\right\rangle$, is given by (Heitsch et al. 2001):

$$
\left\langle B_{o, \perp}\right\rangle^{2}=4 \pi \rho \frac{\sigma_{v_{l o s}}^{2}}{\sigma\left(\tan \delta_{\mathrm{p}}\right)^{2}}
$$

where $\rho$ is the density of the medium, $\theta_{p}$ is the measured polarization position angle, $\left\langle\theta_{p}\right\rangle$ is the weighted mean of the measured position angles, $\delta_{p} \equiv \theta_{p}-\left\langle\theta_{p}\right\rangle$, and $\sigma_{v_{l o s}}$ is the dispersion of the line-of-sight velocity in the medium.

\section{OBSERVATIONS, DATA REDUCTION AND RESULTS}

\subsection{Radio Observations}

RM data were acquired at the Australia Telescope Compact Array (ATCA) over the period 2004 July 10th -18th, using the 6A array configuration spanning baselines from $336.7 \mathrm{~m}$ to $5938.8 \mathrm{~m}$, with a total of 32 adjacent frequency channels each of bandwidth $4 \mathrm{MHz}$ centered on $1384 \mathrm{MHz}$. The standard primary flux calibrator PKS B1934-638, whose flux at $1384 \mathrm{MHz}$ was assumed to be $14.94 \mathrm{Jy}$, was observed at the beginning and the end of each observation. The secondary calibrator PKS B0252-712 was observed every hour and was used to correct for polarization leakages and to calibrate the time-dependent antenna gains. To cover the whole SMC as well as the region around it, we scanned a 40-square-degree region divided into 440 pointings. For each pointing, we obtained 30 cuts of 30 seconds, resulting in a total observing time of 110 hours. These observations have poor sensitivity on scales larger than $\sim 30$ arcseconds. Therefore, extended sources in the SMC and diffuse emission from the SMC itself 
are not detected; what we mainly see are background point sources.

The MIRIAD package was used for data reduction (Sault \& Killeen 2003). Data were first flagged and calibrated. Flagging and rebinning the $324 \mathrm{MHz}$-wide channels resulted in 13 $8-\mathrm{MHz}$ wide channels. For each pointing and frequency channel, maps of Stokes parameters $Q$ and $U$ were made. These maps were then deconvolved using the CLEAN algorithm. A final map was generated by convolving the sky model with a Gaussian beam of dimensions 13 " $\times 8$ ". We produced a restored image for each pointing, for Stokes $Q$ and $U$ at each of the 13 frequency channels. This results in a total of 11440 images, each with a sensitivity of $\sim 1 \mathrm{mJy}$ per beam.

For each pointing and each channel, a linearly polarized intensity (PI) map, corrected for positive bias was made. To ensure that no source was lost through bandwidth depolarization, PI maps over all channels were then averaged together to make a single polarization map for each pointing. A linearly polarized intensity map with sensitivity of $0.4 \mathrm{mJy}$ per beam, covering all 440 pointings, was created using the task LINMOS. Polarized point sources were identified from the mosaicked polarized intensity image using the task SFIND, which implements the False Discovery Rate (FDR) algorithm (Hopkins et al. 2002). These polarized point sources are likely to be extragalactic as their positions do not coincide with known supernova remnants (SNRs) (Filipović et al. 2005). Figure 2 shows two examples of linear polarization detected from extragalactic background sources in the field. For each of the 13 channel maps for each source, values of Stokes $Q$ and $U$ were extracted for the peak pixel and the eight brightest pixels (in polarized intensity) surrounding it.

The RM of each source was computed following the algorithm developed by Brown et al. (2003). As long as the RMs have magnitudes less than $\sim 2700 \mathrm{rad} \mathrm{m}^{-2}$, our data do not suffer from an $\mathrm{n} \pi$ ambiguity because of the closely spaced frequency channels. For each pixel of each source, the RM per pixel was calculated by least-squares fitting the unwrapped polarization position angle (see Brown et al. 2003) as a function of the wavelength squared. Figure 3 shows the least squares fit for one of our background sources. These RMs were then passed through tests to ensure sufficient signal-tonoise and a reasonable quality of fit. A source was accepted if more than half of the pixels yield reliable RMs (quality of fit ${ }^{2} \mathrm{Q}>0.1$ ). The source RM (weighted by the error in the RM for each pixel) and its uncertainty were computed from the good pixels. If the scatter of RM from pixel to pixel within the same source was larger than twice the average statistical error of the source pixels, the source was rejected.

The data reduction procedures described above produce 70 reliable and accurate RMs as listed in Table 3. After comparing catalogued positions of HII regions (Henize 1956) with those of the extragalactic background sources, we find that source 134 has a projection that coincides with N90, an active star forming region in the wing of the SMC. The RM through this particular sight line traces magnetic field and electron distribution through the HII region as well as through the diffuse ISM.

As mentioned in $\S 1.3, \mathrm{RM}_{\text {MilkyWay }}$ can be estimated using the RM values of extragalactic sources whose projected positions lie close to, but outside the SMC. We define the boundary of the SMC to be where the neutral hydrogen col-

\footnotetext{
2 The probability of a random distribution generating a value of $\chi^{2}$ greater than the observed value, for $\nu$ degrees of freedom
}

umn density drops below $2 \times 10^{21}$ atoms $\mathrm{cm}^{-2}$ or the extinction corrected intrinsic $\mathrm{H} \alpha$ intensity of the SMC drops below 25 deci-Rayleigh ( $\mathrm{dR})$, where $1 \mathrm{R}=10^{6}$ photons per $4 \pi$ steradian $=2.42 \times 10^{-7} \mathrm{ergs} \mathrm{cm}^{-2} \mathrm{~s}^{-1} \mathrm{sr}^{-1}$ (see $\S 2.3$ ). A source's projection is considered to be inside the SMC if it lies inside either the HI column density or the $\mathrm{H} \alpha$ threshold. We find that 10 extragalactic sources satisfy this criteria and are indicated with $*$ in Table 3 .

The data are insufficient to constrain a foreground RM dependence on declination as there are very few background sources at more southerly declinations. However, it is obvious that background sources to the west of the SMC have values of RM which are more positive than those to the east, hence, we perform a least square fit to the value of the foreground rotation measure as a function of right ascension in degrees (Figure 4). The best fit has the form

$$
\mathrm{RM}_{\text {MilkyWay }}=(46.1 \pm 4.1)-(4.9 \pm 0.9) \times \mathrm{a} \mathrm{rad} \mathrm{m}^{-2}
$$

where a is the offset in degrees eastward from zero right ascension.

After subtracting the fit to the foreground RM as given in Equation (5) and propagating the associated uncertainties into $\mathrm{RM}_{\mathrm{SMC}}$, the distribution of RM through the SMC is shown in Figure 5 and listed in Table 5. The RMs of the 10 extragalactic sources which lie directly behind the SMC range from $-400 \pm 60 \mathrm{rad} \mathrm{m}^{-2}$ (source 135) to $0 \pm 50 \mathrm{rad} \mathrm{m}^{-2}$ (source 136), with a weighted mean of $-30 \mathrm{rad} \mathrm{m}^{-2}$, a weighted standard deviation (calculated using Equation (4.22) in Bevington \& Robinson (2003)) of $40 \mathrm{rad} \mathrm{m}^{-2}$ and a median of $-75 \mathrm{rad}$ $\mathrm{m}^{-2}$. After the foreground subtraction, RMs of sources whose projections lie outside the SMC should be zero by construction. We find a residual $\mathrm{RM}$ of $0 \mathrm{rad} \mathrm{m}^{-2}$ with a weighted standard deviation of $20 \mathrm{rad} \mathrm{m}^{-2}$.

From the fact that 9 out of 10 extragalactic sources behind the SMC have negative RMs and the other has a RM consistent with zero, we argue that the underlying field is unlikely to be random in direction as this would produce equal numbers of positive and negative RMs across the galaxy with a mean close to zero. If we are observing a random field, the probability of getting at least nine out of ten RMs of the same sign is $0.4 \%$. In other words, the magnetic field across the entire SMC is coherently directed away from us at a $99.6 \%$ confidence level. The measured large RMs through the SMC also cast doubt on the orientation of the plane-of-the-sky magnetic field obtained by Loiseau et al. (1987) and Haynes et al. (1991) from linearly polarized radio synchrotron emission, because our observed mean $\mathrm{RM}$ of $-30 \mathrm{rad} \mathrm{m}^{-2}$ rotates the polarization position angle by $\sim 70^{\circ}$ at $20 \mathrm{~cm}$. Since Loiseau et al. (1987) and Haynes et al. (1991) did not correct for Faraday rotation, their angles do not correspond to intrinsic angles in the SMC.

\subsection{Optical Starlight Polarization Data}

Mathewson \& Ford (1970a) observed 76 stars in the SMC, along with 60 Galactic stars towards the SMC at distances from $50 \mathrm{pc}$ to $2 \mathrm{kpc}$ to correct for the foreground polarization. They found that the foreground signal has a fractional polarization of $0.2 \%$. The distribution of SMC stars and their raw optical polarization position angles are plotted in Figure 1.

As pointed out in $\S 1.4$, the Galactic foreground polarization is directed along the SMC-LMC connection, so a careful foreground correction is required. Schmidt (1976) subdivided the SMC's projection onto the celestial sphere into five regions and calculated the foreground correction for each re- 
gion by studying a large number of Galactic foreground stars at different distances. We have applied this improved Galactic foreground correction to the 76 stars observed by Mathewson $\&$ Ford (1970a). Because of the large angular extent of the SMC, measuring the deviation of polarization position angles with respect to the north is not useful. Instead, we choose our reference direction to be along the great circle joining the SMC and the LMC on the celestial sphere. The positions, polarization position angles and associated errors of the polarization vectors of 76 SMC stars, after foreground subtraction, are listed in Table 6.

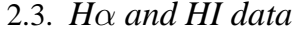

In order to estimate the thermal electron density in the SMC, we have used the continuum subtracted SHASSA $\mathrm{H} \alpha$ map of the SMC smoothed to 4 arcminutes (Gaustad et al. 2001). The image has a sensitivity of $5 \mathrm{dR}$.

Knowing the $\mathrm{H} \alpha$ intensity of the SMC and the foreground extinction allows one to evaluate the emission measure, as will be shown in $\S 3.2$. To correct the observed $\mathrm{H} \alpha$ intensity for interstellar extinction, we use the integrated neutral hydrogen (HI) column density map of the SMC presented by Stanimirović et al. (1999) from ATCA and Parkes spectral line observations. The column density was derived by integrating the $21 \mathrm{~cm} \mathrm{HI} \mathrm{signal} \mathrm{over} \mathrm{the} \mathrm{heliocentric} \mathrm{velocity} \mathrm{range}+90$ to $+215 \mathrm{~km} \mathrm{~s}^{-1}$, and the resulting column density map has an angular resolution of 1.6 arcminutes.

\section{THE LINE-OF-SIGHT MAGNETIC FIELD STRENGTH IN THE} WARM IONIZED MEDIUM OF THE SMC

In this section, we construct three ionized gas models from the extinction corrected $\mathrm{H} \alpha$ intensity of the SMC and from pulsar dispersion measures. These models allow us to estimate the average magnetic field strength along the line of sight, $\overline{B_{\|}}$, from the RMs presented in $\S 2.1$.

\subsection{Pulsar Dispersion Measure and Rotation Measure}

The dispersion measure (DM) of a pulsar is an integral of the electron density content along the line of sight, defined as:

$$
\mathrm{DM}=\int_{0}^{L} n_{e}(l) d l=\overline{n_{e}} L \mathrm{pc} \mathrm{cm}{ }^{-3}
$$

where $\overline{n_{e}}$ is the average electron density along the total path length $L$. There are 5 known radio pulsars in the SMC. Their positions, measured DMs and an RM for the one source with Faraday rotation information, are listed in Table 4.

We subtract the Galactic contribution to DMs of SMC pulsars using the NE2001 Galactic free electron model developed by Cordes \& Lazio (2002). The average DM of pulsars in the SMC after the removal of the Galactic contribution is $\left\langle\mathrm{DM}_{\mathrm{SMC} \text {,pulsar }}\right\rangle=80.9 \mathrm{pc} \mathrm{cm}^{-3}$. If we assume that the pulsars are evenly distributed through the SMC, the total DM through the $\mathrm{SMC}$ is approximately twice the mean value, that is, $\left\langle\mathrm{DM}_{\mathrm{SMC}}\right\rangle \approx 162 \mathrm{pc} \mathrm{cm}^{-3}$.

Following the treatment of Manchester et al. (2006), the mean electron density $\left\langle n_{e}\right\rangle$ in the SMC can be estimated by computing the dispersion of DMs and the dispersion of pulsars' spatial coordinates. The underlying assumption is that the SMC is spherically symmetric. We assume that the mean distance to the SMC pulsars is $60 \mathrm{kpc}$, instead of $50 \mathrm{kpc}$ as in Manchester et al. (2006), and that the offsets of pulsar locations in RA and DEC directions are independent. The mean electron density in the SMC is given by

$$
\left\langle n_{e}\right\rangle=\frac{\sigma_{\mathrm{DM}}}{\sigma_{\text {spatial }, 1 \mathrm{D}}}
$$

where $\sigma_{\mathrm{DM}} \approx 48 \mathrm{pc} \mathrm{cm}^{-3}$ is the dispersion of pulsar DMs, after foreground subtraction; and $\sigma_{\text {spatial, } 1 \mathrm{D}} \approx 1230 \mathrm{pc}$ is the one-dimensional spatial dispersion of their positions. This estimation gives a mean electron density of $\left\langle\mathrm{n}_{e}\right\rangle \approx 0.039 \mathrm{~cm}^{-3}$ in the SMC.

Out of the 5 known radio pulsars in the SMC, only one (PSR J0045-7319) has a measured rotation measure, with a value of $-14 \pm 27 \mathrm{rad} \mathrm{m}^{-2}$ (Crawford et al. 2001). Comparing the DM of this pulsar from Table 4 with $\left\langle\mathrm{DM}_{\mathrm{SMC}}\right\rangle$, one can conclude that this pulsar is located approximately half way through the galaxy. Following the foreground subtraction procedure described in $\S 2.1$, the component of the RM from this pulsar that results from the magnetized medium in the SMC is $-40 \pm$ $30 \mathrm{rad} \mathrm{m}^{-2}$. This negative value is consistent with negative signs of RMs of extragalactic sources through the SMC as given in Table 5.

\subsection{Emission Measure}

The emission measure (EM) of ionized gas along the line of sight is defined as

$$
\mathrm{EM}=\int_{0}^{L} n_{e}(l)^{2} d l=\overline{n_{e}^{2}} L \mathrm{pc} \mathrm{cm}^{-6}
$$

where $\overline{n_{e}^{2}}$ is the average of the square of the electron density along the total path length $L$.

We derive an emission measure map of the SMC from the smoothed and star-subtracted $\mathrm{H} \alpha$ emission in this region (Gaustad et al. 2001) by correcting for both foreground extinction caused by dust in the Milky Way and internal extinction in the SMC. The foreground Milky Way contribution to the observed $\mathrm{H} \alpha$ emission is estimated by the off source $\mathrm{H} \alpha$ intensity in regions surrounding the SMC. We assume a constant Galactic foreground HI column density of (4.3 \pm $1.3) \times 10^{20}$ atoms $\mathrm{cm}^{-2}$ (Schwering \& Israel 1991) and a dimensionless Galactic dust-to-gas ratio $k$ of 0.78 (Pei 1992), where $k$ is defined as:

$$
k \equiv 10^{2} 1\left(\tau_{\mathrm{B}} / N_{\mathrm{HI}}\right) \quad \mathrm{cm}^{-2}
$$

where $\tau_{B}$ denotes the optical depth in the optical $B$ band and $N_{\mathrm{HI}}$ denotes the neutral hydrogen column density. For the internal extinction of the SMC, the correction is derived from the HI column density map (Stanimirović et al. 2004) and a dust-to-gas ratio $k$ of 0.08 (Pei 1992). We have used the empirical extinction curves of the Milky Way and the SMC at the wavelength of $\mathrm{H} \alpha\left(\lambda_{\mathrm{H} \alpha}=6563 \mathrm{~A}\right)$

$$
\xi\left(\lambda_{\mathrm{H} \alpha}\right)=\tau_{\mathrm{H} \alpha} / \tau_{\mathrm{B}}=0.6
$$

(Pei 1992), where $\tau_{\mathrm{H} \alpha}$ is the optical depth at 6563A. The optical depth at the wavelength of $\mathrm{H} \alpha$ can thus be expressed as

$$
\tau_{\mathrm{H} \alpha}=k\left(N_{\mathrm{HI}} /\left(10^{21} \mathrm{~cm}^{-2}\right)\right) \xi\left(\lambda_{\mathrm{H} \alpha}\right)
$$

The intrinsic $\mathrm{H} \alpha$ intensity of the SMC is calculated assuming $^{3}$ that the $\mathrm{H} \alpha$ emitting gas is uniformly mixed with dust in a region of optical depth $\tau_{\mathrm{H} \alpha}$. The EM for $\mathrm{H} \alpha$ intensity

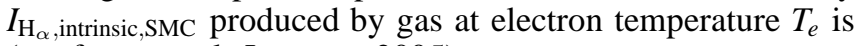
(see for example Lequeux 2005):

$$
\mathrm{EM}=\frac{I_{\mathrm{H}_{\alpha}, \text { intrinsic }, \mathrm{SMC}}\left(T_{e} / 10000 \mathrm{~K}\right)^{0.5}}{0.39\left(0.92-0.34 \ln \left(T_{e} / 10000 K\right)\right)}
$$

where $\mathrm{T}_{e}$ is the electron temperature of the diffused ionized medium in the SMC and $I_{\mathrm{H}_{\alpha}}$,intrinsic,SMC in Rayleighs is the intrinsic $\mathrm{H} \alpha$ intensity of the SMC .

\footnotetext{
${ }^{3}$ See Appendix A for details
} 
As no measurement of the temperature of SMC's diffuse ionized medium exists in the literature, we estimate $T_{e}$ by adding $2,000 \mathrm{~K}$ to the average temperature in HII regions $(\sim$ 12,000K (Dufour \& Harlow 1977)) in the SMC, by analogy with the diffused ionized medium in the Milky Way, which are $\sim 2,000 \mathrm{~K}$ hotter than Galactic HII regions (Madsen et al. 2006). We thus adopt $\mathrm{T}_{e} \sim 14,000 \mathrm{~K}$. The resulting emission measure map is shown in Figure 6.

\subsection{Diffuse Ionized Gas Models}

Our models are based on the assumption that there is no correlation between the fluctuations in the electron density and in the magnetic field. From Equation (2) the average magnetic field strength along the line of sight $\overline{B_{\|}}$is then:

$$
\overline{B_{\|}}=\frac{\mathrm{RM}_{\mathrm{SMC}}}{0.812 \overline{n_{e}} L}
$$

For gas densities lower than $10^{3} \mathrm{~cm}^{-3}$, there is no observational evidence of correlation between the magnetic field strength and gas density (Crutcher et al. 2003). As discussed by Beck et al. (2003), if pressure equilibrium is maintained, one expects an anti-correlation between the magnetic field and the thermal electron density on small scales. This would lead to an underestimation of $\overline{B_{\|}}$. On the other hand, $n_{e}$ and $B_{\|}$ might be correlated by compression in SNR shocks in selected regions. This would lead to an overestimation of $\overline{B_{\|}}$. Depending on the property of the turbulent ISM in the SMC, our estimates of $\overline{B_{\|}}$presented in the following are potentially subject to systematic bias by a factor of two to three.

The following models ignore the presence of individual HII regions and only focus on diffused ionized regions. As mentioned in $\S 2.1$, source 134 appears to coincide with N90, an active star formation region in the SMC. Estimations of $\overline{B_{\|}}$ in the following models along this particular sight line might not reflect the true value and it is excluded when calculating the average line-of-sight magnetic field strength of the SMC, $\left\langle B_{c, \|}\right\rangle$.

\subsubsection{Model 1: Constant Dispersion Measure}

We can estimate the line of sight magnetic field strength $\left(\overline{B_{\|}}\right)$by assuming that the dispersion measure through the SMC is constant across the galaxy, with $\left\langle\mathrm{DM}_{\mathrm{SMC}}\right\rangle=\overline{n_{e}} L=$ $162 \mathrm{pc} \mathrm{cm}^{-3}$. Combining Equations (6) and (13), we find

$$
\overline{B_{\|}}=\frac{\mathrm{RM}_{\mathrm{SMC}}}{0.812\left\langle\mathrm{DM}_{\mathrm{SMC}}\right\rangle}
$$

Estimations of $\overline{B_{\|}}$obtained using this model are listed in the second column of Table 7 . The weighted magnetic field along the line of sight averaged across the SMC is $-0.20 \mu \mathrm{G}$. This model gives crude estimates of $\overline{B_{\|}}$, since in reality, both the depth of the SMC $(L)$ and the line of sight average of electron density $\overline{n_{e}}$ vary from one sight line to the other, while their product need not stay the same.

\subsubsection{Model 2: Constant $\overline{n_{e}}$, varying $L$}

The motivation behind this model is the observational evidence for the variation of the line-of-sight depth as a function of position in the SMC, as seen in both the HI velocity dispersion (Stanimirović et al. 2004) and the variation of the distance modulus to Cepheid variables (Lah et al. 2005) across the SMC.
It is useful to define filling factor $f$, the fraction of the total path length occupied by thermal electrons, as the following (see for example Reynolds 1991; Berkhuijsen et al. 2006)

$$
f=\frac{\overline{n_{e}}}{\overline{n_{\text {cloud }}}}
$$

where $\overline{n_{\text {cloud }}}$ is the average electron density in ionized gas clouds along the line of sight and $\overline{n_{e}}$ is the mean electron density along the line of sight. For the special case where the electron densities in the individual ionized gas clouds along the line of sight are the same, the filling factor can be expressed as

$$
f=\frac{{\overline{n_{e}}}^{2}}{\overline{n_{e}{ }^{2}}}
$$

In this model, we assume that the filling factor $f$, and the mean electron density along the line of sight $\overline{n_{e}}$ remain the same across the galaxy, while the depth $L$ of the SMC varies. Additionally, we assume that all ionized gas clouds along the line of sight have the same electron density $n_{\text {cloud }}$. Using the definition of the filling factor $f$ given in Equations 15 and 16, one can express the average DM and EM through the SMC as

$$
\begin{aligned}
& \left\langle\mathrm{DM}_{\mathrm{SMC}}\right\rangle=\overline{n_{e}}\left\langle L_{\mathrm{SMC}}\right\rangle=\overline{n_{\text {cloud }}} f\left\langle L_{\mathrm{SMC}}\right\rangle=n_{\text {cloud }} f\left\langle L_{\mathrm{SMC}}\right\rangle \\
& \left\langle\mathrm{EM}_{\mathrm{SMC}}\right\rangle=\overline{n_{e}^{2}}\left\langle L_{\mathrm{SMC}}\right\rangle=\overline{n_{\text {cloud }}^{2}} f\left\langle L_{\mathrm{SMC}}\right\rangle=n_{\text {cloud }}^{2} f\left\langle L_{\mathrm{SMC}}\right\rangle
\end{aligned}
$$

where $\left\langle L_{\mathrm{SMC}}\right\rangle$ denotes the average depth of the SMC. Combining the two equations above, we obtain

$$
n_{\text {cloud }}=\frac{\left\langle\mathrm{EM}_{\mathrm{SMC}}\right\rangle}{\left\langle\mathrm{DM}_{\mathrm{SMC}}\right\rangle}
$$

We assumed in the previous sections that $\left\langle\mathrm{DM}_{\mathrm{SMC}}\right\rangle=162$ $\mathrm{pc} \mathrm{cm}^{-3} \cdot\left\langle\mathrm{EM}_{\mathrm{SMC}}\right\rangle$ is estimated by the average emission measure through the SMC defined by the HI column density contour in Figure 5. Individual HII regions with intrinsic $\mathrm{H} \alpha$ intensity higher than $500 \mathrm{dR}$ are masked out before taking the average, resulting in $\left\langle\mathrm{EM}_{\mathrm{SMC}}\right\rangle \approx 16 \mathrm{pc} \mathrm{cm}^{-6}$. This yields a mean density in an ionized cloud $n_{\text {cloud }} \approx 0.10 \mathrm{~cm}^{-3}$. The filling factor $f$ is estimated using Equation 15 assuming a mean electron density $\overline{n_{e}}=\left\langle n_{e}\right\rangle \approx 0.039 \mathrm{~cm}^{-3}$ obtained from the pulsar DM analysis in $\S 3.1$, to yield $f=0.39$. Using the definition of filling factor given in Equation 16, we can express the EM towards an extragalactic background source as

$$
\mathrm{EM}_{\text {source }}=n_{\text {cloud }}^{2} f L_{\text {source }}
$$

Solving for $L_{\text {source }}$, the path length through the SMC to an extragalactic source, yields

$$
L_{\text {source }}=\frac{\mathrm{EM}_{\text {source }}}{f n_{\text {cloud }}{ }^{2}}
$$

Using the above equation, we have computed the average path length through the SMC to the extragalactic sources to be $\langle L\rangle$ $\approx 10 \mathrm{kpc}$ with a standard deviation $\Delta L \approx 6 \mathrm{kpc}$, which is roughly consistent with Stanimirović et al. (2004).

Using additional information from $\mathrm{H} \alpha$, the mean magnetic field strength parallel to the line of sight can be found by

$$
\overline{B_{\|}}=\frac{\mathrm{RM}_{\mathrm{SMC}}}{0.812\left\langle\mathrm{DM}_{\mathrm{SMC}}\right\rangle}\left(\frac{\left\langle\mathrm{EM}_{\mathrm{SMC}}\right\rangle}{\mathrm{EM}_{\text {source }}}\right)
$$

This equation has a similar form to Equation (14) with an additional correction factor for the variation of EM across the galaxy. Values for $\overline{B_{\|}}$using this estimate are listed in the third column of Table 7 . The weighted magnetic field along the line of sight averaged across the SMC is $-0.16 \mu \mathrm{G}$. 


\subsubsection{Model 3: Constant Occupation Length $f L$}

In this model, we assume that the occupation length $f L$ (the effective length occupied by thermal electrons along a sight line) is constant while the line-of-sight mean electron density $\overline{n_{e}}$ is allowed to vary between sight lines. In addition, we assume that $n_{\text {cloud }}$, the density of all ionized clouds along the line of sight, is the same. One can manipulate Equations (17) $\&$ (18) and solve for the occupation length $f L$ :

$$
f L=\frac{\left\langle\mathrm{DM}_{\mathrm{SMC}}\right\rangle^{2}}{\left\langle\mathrm{EM}_{\mathrm{SMC}}\right\rangle}
$$

We found in $\S 3.1$ that $\left\langle\mathrm{DM}_{\mathrm{SMC}}\right\rangle=162 \mathrm{pc} \mathrm{cm}^{-3} .\left\langle\mathrm{EM}_{\mathrm{SMC}}\right\rangle \approx$ $16 \mathrm{pc} \mathrm{cm}^{-6}$ was estimated in $\S 3.3 .2$. This yields an occupation length $f L \approx 1.6 \mathrm{kpc}$.

Using the definition of filling factor given in Equation 16, we can express EM towards an extragalactic background source as

$$
\mathrm{EM}_{\text {source }}=\overline{n_{e}^{2}} L={\overline{n_{e}}}^{2} L / f
$$

Solving for $\overline{n_{e}}$ yields

$$
\overline{n_{e}}=\sqrt{\frac{\mathrm{EM}_{\text {source } f}}{L}}
$$

Substituting the above expressions into Equation (13) and solving for the mean magnetic field strength through different sight lines gives

$$
\overline{B_{\|}}=\frac{\mathrm{RM}_{\mathrm{SMC}}}{0.812\left\langle\mathrm{DM}_{\mathrm{SMC}}\right\rangle} \sqrt{\frac{\left\langle\mathrm{EM}_{\mathrm{SMC}}\right\rangle}{\mathrm{EM}_{\text {source }}}}
$$

This equation has a similar form to Equation (22), but with a square root rather than linear dependence on EMs. Values of $\overline{B_{\|}}$derived using this method are listed in the fourth column of Table 7. The weighted magnetic field along the line of sight averaged across the SMC is $-0.19 \mu \mathrm{G}$.

\subsubsection{Summary of the Models}

All models yield field strengths that are mostly consistent with each other within their uncertainties. Model 1 is a simplified picture of the physical situation which does not make use of all the known information, and thus it provides rough estimates of $\bar{B}_{\|}$. Model 2 and 3 are more sophisticated, and thus they provide estimates that probably better describe the true $\bar{B}_{\|}$. Out of the three models, model 2 makes use of the most information one can get from pulsar dispersion measure analysis and the $\mathrm{H} \alpha$ intensity of the SMC. Model 3 has the most degrees of freedom: both $f$ and $L$ are allowed to vary from one line of sight to the other as long as their product stays the same, and the average electron density along the line of sight $\overline{n_{e}}$ is allowed to change depending on which sight line one is looking through. However, one should note that both model 2 and 3 assume that the electron density in ionized clouds along the line of sight is either $n_{\text {cloud }}$ (a constant) or 0 , that is, a smooth fluctuation of electron density along the line of sight is forbidden. The following discussions refer to estimations from model 3 unless specified otherwise.

The 10 extragalactic sources that lie behind the SMC yield line-of-sight magnetic field strengths ranging from $-1.5 \pm 0.6$ (source 135) to $0.0 \pm 0.2 \mu \mathrm{G}$ (source 136), where the negative sign denotes a magnetic field directed away from us. Only three out of the 10 sources have a $\bar{B}_{\|}$consistent with zero, while all the others are negative at at least the $2 \sigma$ and four are negative at the $3 \sigma$ confidence level. The distribution of the magnetic field through the SMC is plotted in Figure 6 on the emission measure map of the galaxy. The weighted mean of the line-of-sight strength of the magnetic field is $-0.19 \mu \mathrm{G}$, which in subsequent discussion we adopt as the coherent magnetic field strength of the SMC parallel to the line of sight, $\left\langle B_{c, \|}\right\rangle$.

Using the standard error in the weighted mean prescription in Cochran (1977) and assuming that there is one overall underlying field in the SMC; we find that $\left\langle B_{c, \|}\right\rangle=-0.19 \mu \mathrm{G}$ with a standard error in the weighted mean of $0.06 \mu \mathrm{G}$. We can also quantify the scatter of the field by quoting at $68 \%$ confidence level that the coherent magnetic field strength of the SMC, $\left\langle B_{c, \|}\right\rangle$ is $-0.2_{-0.3}^{+0.1} \mu \mathrm{G}$. Note that the strength of the magnetic field that we derive in this section is independent of the temperature of the WIM one picks to convert the $\mathrm{H} \alpha$ intensity into an EM (see Equation 12) because the expressions of the field strength (Equation 14, $22 \& 26$ ) only involve the ratio of emission measures.

\section{THE PLANE-OF-THE-SKY MAGNETIC FIELD OF THE SMC}

\subsection{Estimation of $\left\langle B_{o, \perp}\right\rangle$ using the $C-F$ method}

In $\S 3$, we have derived the line-of-sight magnetic field strength of ionized gas in the SMC using the RMs of extragalactic radio sources. Now, we would like to estimate the strength of the magnetic field perpendicular to the line of sight by applying the C-F method to the starlight polarization data presented in $\S 2.2$. Since the infrared dust emission of the SMC has a similar morphology to its $\mathrm{H} \alpha$ intensity, we assume that dust is in the warm ionized medium (WIM) of the SMC. In addition, Rodrigues et al. (1997) found, from analysis of extinction and polarization data of SMC stars, that the SMC has smaller grain sizes than those in the Milky Way (where dust lie mostly in the warm neutral medium (WNM), see Lockman \& Condon (2005)). One expects smaller dust grains in the WIM than in the WNM due to grain shattering and grain-grain collisions (Jones et al. 1996). The fact that the SMC has smaller grain sizes than those in the Milky Way further supports our assumption that dust is in the WIM of the SMC. To estimate the strength of the plane-of-the-sky component of field, we use Equation (4). The density of the medium, $\rho$, is

$$
\rho=\gamma_{H} n_{H} m_{H}
$$

where $\gamma_{H} \approx 1.22$ is the equivalent molecular weight of the ISM for SMC abundances (Russell \& Dopita 1992; Peimbert $\&$ Peimbert 2000), $n_{H}$ is the number density of hydrogen and $m_{H}$ is the mass of a hydrogen atom. We assume that at a temperature of $14,000 \mathrm{~K}$, hydrogen in the WIM is completely ionized, with a negligible ionization fraction for heavier elements. Hence, the mean ISM hydrogen number density is $n_{H}=n_{\text {cloud }} \sim 0.1 \mathrm{~cm}^{-3}$ (see $\S 3.3 .2$ ). The average HI line-ofsight velocity dispersion is $22 \pm 2 \mathrm{~km} \mathrm{~s}^{-1}$ (Stanimirović et al. 2004), which we adopt as the line-of-sight velocity dispersion in the WIM also.

To estimate the ordered field strength in the plane of the sky, we eliminate stars with large uncertainty in the polarized fraction as well as stars that lie outside the main body of the SMC as defined by the column density contour value of $2 \times 10^{21}$ atoms cm$~^{-2}$ (see Figure 5). The starlight polarization measurements that are used in this calculation are listed in Table 6 . The average polarization position angle $\left\langle\theta_{p}\right\rangle$ deviates $+4^{\circ} \pm 12^{\circ}$ from the great circle joining the SMC to the LMC, measured counterclockwise. The standard deviation of $\tan \delta_{p}$ 
is then calculated. Using Equation (4), the ordered component of the magnetic field in the plane of the sky averaged over the whole SMC is $\left\langle B_{o, \perp}\right\rangle=1.6 \pm 0.4 \mu \mathrm{G}$. We choose not to break the SMC up into sub-regions and estimate the plane-of-thesky field in each because there are not a sufficient number of polarization measurements in each sub-region to sensibly estimate $\left\langle\theta_{p}\right\rangle$ and $\sigma\left(\tan \theta_{p}\right)$. This analysis is complementary to the rotation measure study since it provides information on the ordered component of the plane-of-the-sky magnetic field. We assume that the fields obtained using the C-F method and the RM method are orthogonal components of the same large scale field, so for a magnetic field whose line-of-sight component is coherent and whose plane-of-the-sky component is ordered, the 3D magnetic field vector is likely to be coherent as well. Hence, we write $\left\langle B_{c, \perp}\right\rangle=\left\langle B_{o, \perp}\right\rangle$.

However, this calculation is subjected to various uncertainties. In the Milky Way, it is found that dust exists mainly in the WNM (Lockman \& Condon 2005) but WIM dust emission has also been detected (Lagache et al. 1999). In the above calculation, we have assume that all dust lie in SMC's WIM. In reality, some dust must be present in the WNM of the SMC (due to the correlation of IR dust emission and HI column density, see Stanimirović et al. (2000)) and hence when estimating $\rho$ and $\sigma_{v_{\text {los }}}$ in the SMC, one should take into account of the contribution from the neutral medium as well as the ionized medium. Also, if the polarization measurements are from stars on the near side of the SMC, we are merely probing the "surface" magnetic field of the galaxy (Magalhães et al. 1990). Furthermore, since dust regions entrenched in oppositely directed magnetic fields would polarize starlight in the same fashion, the plane-of-the-sky magnetic field strength derived is correct only if the ordered magnetic field direction does not change appreciably along the entire line of sight. We will overestimate the field strength when the plane-of-the-sky field reverses direction along the line of sight. A correction factor was introduced by Myers \& Goodman (1991) to account for this effect. We do not need to correct for this here if the $\mathrm{C}-\mathrm{F}$ method and the RM method probe the same large scale field, since RM data demonstrate that the field does not reverse on large scales along the line-of-sight.

\subsection{Estimation of $\left\langle B_{\text {total }, \perp}\right\rangle$}

We now compute the total plane-of-the-sky (i.e. random and ordered fields combined) magnetic field strength $\left\langle B_{\text {total }, \perp}\right\rangle$ using equipartition energy arguments. If we assume that the cosmic ray energy density is the same as the magnetic field energy density, one can estimate $\left\langle B_{\text {total }, \perp}\right\rangle$ using the relations given in Pacholczyk (1970) and Melrose (1980) between the specific intensity of synchrotron emission, the total plane-of-the-sky field and the synchrotron emitting path length through the galaxy. We assume that the synchrotron emitting layer of the SMC has the same thickness as the Faraday rotating layer, i.e. $L_{\text {synchrotron }}=\langle L\rangle \approx 10 \mathrm{kpc}(\$ 3.3 .2)$. Using a spectral index $\alpha=0.87$, a cosmic ray energy density $K=5 \times 10^{-17} \mathrm{erg}^{-1} \mathrm{~cm}^{-3}$ (Beck 1982), and a non-thermal intensity $I_{\nu}=6.4 \times 10^{-20} \mathrm{erg} \mathrm{s}^{-1} \mathrm{~cm}^{-2} \mathrm{~Hz}^{-1} \mathrm{sr}^{-1}$ at $\nu=2.3 \mathrm{GHz}$ (Loiseau et al. 1987), we obtain $\left\langle B_{\text {total }, \perp}\right\rangle \approx 2.2 \mu \mathrm{G}$.

As pointed out by Beck \& Krause (2005), the above calculation is likely to underestimate $\left\langle B_{\text {total }, \perp}\right\rangle$ due to the uncertainty in $K$, and the integration of the radio spectrum over a fixed frequency range (instead of a fixed energy range to approximate the cosmic ray spectrum). We have used the revised equipartition estimate of the magnetic field given in Beck \& Krause (2005) to compute $\left\langle B_{\text {total }, \perp}\right\rangle$. Using the ratio of num- ber densities of protons to electrons for cosmic rays accelerated in SNRs $K_{0} \approx 100$ and $L_{\text {synchrotron }} \approx 10 \mathrm{kpc}$, we obtain an equipartition field strength $\left\langle B_{\text {total }, \perp}\right\rangle=3.2 \mu \mathrm{G}$.

\subsection{The Random Magnetic Field in the SMC}

The random components of the magnetic fields in the Milky Way and in the LMC are found to dominate over the ordered components (Beck 2000; Gaensler et al. 2005). From the dispersion of RMs in the SMC, one can estimate the strength of the random component of the magnetic field ${ }^{5}$.

To allow comparison of the random field derived by combining the synchrotron intensity and starlight polarization measurements (see the next paragraph), which has the same assumptions as ionized gas model 2 in $\S 3.3 .2$, we construct the random magnetic field model of the SMC based on the same ionized gas model. We assume that the average electron density along the line of sight, $\overline{n_{e}}$, is the same through all lines of sight but that the depth of the SMC, $L$, changes from one sight line to another. We decompose the magnetic field along each sight line into coherent and random components, such that the coherent component does not vary across the SMC; the differences between the magnetic field strengths along different sight lines are only due to the random component. In Appendix B we show that the corresponding dispersion in RM is:

$$
\sigma_{\mathrm{RM}}=0.812 l_{o} \overline{n_{e}} \sqrt{\left\langle B_{c, \|}\right\rangle^{2}\left(\frac{\Delta L}{l_{o}}\right)^{2}+B_{r}^{2}\left(\frac{\langle L\rangle}{3 l_{o}}\right)}
$$

where $\sigma_{\mathrm{RM}} \sim 40 \mathrm{rad} \mathrm{m}^{-2}$ is the weighted standard deviation in $\mathrm{RM}$ for the extragalactic sources that lie behind the SMC; $l_{o} \sim$ $90 \mathrm{pc}$ is the typical cell size along the line of sight, which we take to be similar to that in the LMC (Gaensler et al. 2005); $\overline{n_{e}}$ $=0.039 \mathrm{~cm}^{-3}$, is the mean electron density in the SMC as derived in $\S 3.1,\left\langle B_{c, \|}\right\rangle \approx-0.16 \mu \mathrm{G}$ is the average SMC coherent field strength along the line of sight as obtained using ionized gas model $2 ;\langle L\rangle \approx 10 \mathrm{kpc}$ is the average depth of the SMC along different sight lines; and $\Delta L \approx 6 \mathrm{kpc}$ is the standard deviation of the depth of the SMC between different sight lines (see $\S 3.3 .2$ ). Using the above method, we find $B_{r}=19 / \sqrt{l_{0}}$ $\sim 2 \mu \mathrm{G}$. Therefore, in the SMC, the random component of the magnetic field dominates over the coherent magnetic field along the line of sight.

A key prediction of our assumption that the RMs, optical starlight polarization and synchrotron intensity probe different projections of the same large-scale magnetic field is that the independently derived measurements of the random magnetic field must agree. Since the total synchrotron intensity probes the total magnetic field in the plane of the sky while the $\mathrm{C}-\mathrm{F}$ method probes the ordered component in the plane of the sky, one can write:

$$
\left\langle B_{\text {total }, \perp}^{2}\right\rangle=\left\langle B_{o, \perp}^{2}\right\rangle+\left\langle B_{r, \perp}^{2}\right\rangle
$$

where $\left\langle B_{r, \perp}^{2}\right\rangle$ is the random magnetic field strength in the plane of the sky. If we assume that the random field is isotropic, then its strength is given by

$$
B_{r}^{2}=\frac{3}{2}\left\langle B_{r, \perp}^{2}\right\rangle
$$

5 If there is no random field and the uniform component is coherent throughout the galaxy, there will still be an RM gradient across the galaxy due to projection onto the curved celestial sphere. We ignore this small effect and assume that the patch of celestial sphere towards the SMC is flat. 
Using $\left\langle B_{\text {total }, \perp}\right\rangle \approx 3.2 \mu \mathrm{G}$ (see $\S 4.2$ ) and $\left\langle B_{o, \perp}\right\rangle \approx 1.6 \mu \mathrm{G}$ (see $\S 4.1$ ) leads to a random magnetic field strength of $\sim$ $3.4 \mu \mathrm{G}$. Since the estimate of the random field strength using the scatter of rotation measure agrees well with that obtained by combining the synchrotron intensity and starlight polarization measurements, our data demonstrate that our underlying assumptions are self-consistent.

\section{THE 3D MAGNETIC FIELD STRUCTURE OF THE SMC}

We can combine the results of the RM study ( $\$ 3$ ) and of the C-F method ( $\$ 4.1)$ to construct a 3D magnetic field vector for the SMC, assuming that the two methods probe the same field (in terms of strength, overall geometry and fluctuations).

The strength of the coherent magnetic field in the SMC is

$$
B_{\text {total }, c}=\sqrt{\left\langle B_{c, \|}\right\rangle^{2}+\left\langle B_{c, \perp}^{2}\right\rangle}=1.7 \pm 0.4 \mu G
$$

where $\left\langle B_{c, \|}\right\rangle=-0.19 \pm 0.06 \mu \mathrm{G}$ and $\left\langle B_{c, \perp}\right\rangle \approx 1.6 \pm 0.4 \mu \mathrm{G}$ denote the coherent fields found from Faraday rotation and optical starlight polarization, respectively. The three dimensional field is almost entirely in the plane of the sky.

In order to more precisely determine the direction of the coherent magnetic field in the SMC, we need to transform into a cartesian coordinate system with the center of the SMC at the origin. We define our coordinate system such that the $x-y$ plane is the sky plane, the negative $x$ axis points towards the LMC's projection onto the sky plane, and the positive $z$ axis points along the vector joining the center of the SMC to the observer. In this coordinate system, the earth is located at $(0,0,60) \mathrm{kpc}$ and the LMC is located at $(-17,0,13) \mathrm{kpc}$. At the center of the SMC, the line-of-sight magnetic field is in the negative $z$ direction and has a strength of $0.19 \pm 0.06 \mu \mathrm{G}$, while the plane-of-the-sky magnetic field, with a magnitude of $1.6 \pm 0.4 \mu \mathrm{G}$, makes an angle $4^{\circ}$ (counterclockwise) with the positive $x$ axis as shown in $\S 4.1$. Taking into account the ambiguity of the magnetic field direction in the plane of the sky, the coherent magnetic field vector in the SMC could be either

$$
\overrightarrow{B_{c, 1}}=1.6 \hat{x}+0.1 \hat{y}-0.19 \hat{z} \quad \mu \mathrm{G}
$$

or

$$
\overrightarrow{B_{c, 2}}=-1.6 \hat{x}-0.1 \hat{y}-0.19 \hat{z} \quad \mu \mathrm{G}
$$

Equations (32) \& (33) allow us to compute the possible angles that the magnetic field vector makes with the characteristic axes of the Magellanic System. We consider two such axes: that defined by the path from the LMC along the Magellanic Bridge to the SMC, and that defined by the normal to the SMC disk.

Since the 3D structure of the Magellanic Bridge is not well known, we here assume that the Bridge is parallel to $\vec{C}$, the vector which runs from the center of the SMC to that of the LMC. $\vec{C}$ lies in the $x-z$ plane and has the form:

$$
\vec{C}=17 \hat{x}-13 \hat{z} \quad \mathrm{kpc}
$$

This is a crude approximation, since the interaction between the Magellanic Clouds most likely does not follow a straight line.

Separately, the normal to the plane of the SMC's disk is given by the unit vector $\hat{n}$ :

$$
\hat{n}=-0.62 \hat{x}-0.16 \hat{y}-0.77 \hat{z}
$$

Note that the angle between $\vec{C}$ and $\hat{n}$ is $92^{\circ}$ (i.e., the SMC disk is inclined by $2^{\circ}$ degrees from the SMC-LMC axis).
We now consider the extent to which each of $\vec{B}_{c, 1}$ and $\vec{B}_{c, 2}$ are aligned with $\vec{C}$ or are normal to $\hat{n}$. In the following discussion, we quote $90 \%$ confidence intervals in the statistical uncertainties in angles. We consider any angle between vectors of less than $20^{\circ}$ to represent broad alignment, and angles in the range $70^{\circ}-110^{\circ}$ to indicate rough perpendicularity (reflecting the additional systematic uncertainties in our estimates of $\vec{C}$ and $\hat{n}$ ).

We find that the angle between $\vec{B}_{c, 1}$ and $\vec{C}$ is $31_{-5^{\circ}}^{\circ+8^{\circ}}$. We have used Monte Carlo simulations with 50,000 random samplings to delineate the full probability distribution and find that the angle between $\vec{B}_{c, 1}$ and $\vec{C}$ is consistent with alignment within 2.6 $\sigma$. On the other hand, $\vec{B}_{c, 2}$ makes an angle $136_{-8^{\circ}}^{\circ+4^{\circ}}$ with $\vec{C}$. Monte Carlo simulations as described above show that any alignment between $\vec{B}_{c, 2}$ and $\vec{C}$ is ruled out at $>3.1 \sigma$.

Comparing the magnetic field vectors with $\hat{n}$, we find an angle between $\vec{B}_{c, 1}$ and $\hat{n}$ of $123_{-8^{\circ}}^{\circ+4^{\circ}}$. We have used Monte Carlo simulations to find that the angle between $\vec{B}_{c, 1}$ and $\hat{n}$ is consistent with $90^{\circ}$ at $\sim 2.4 \sigma$. The angle between $\vec{B}_{c, 2}$ and $\hat{n}$ is $44_{-5^{\circ}}^{\circ+7^{\circ}}$. Monte Carlo simulations rule out any perpendicularity between $\vec{B}_{c, 2}$ and $\hat{n}$ at $4.2 \sigma$.

The above calculations show that while at $90 \%$ confidence level the SMC magnetic field vector does not orient itself either with the Magellanic Bridge or with the SMC disk, at a slightly higher confidence, the vector $\vec{B}_{c, 1}$ does indeed align with both the Bridge and the disk. We thus favor $\vec{B}_{c, 1}$ as the more likely true magnetic field vector of the SMC over $\vec{B}_{c, 2}$. In this case, the possible alignment between $\vec{B}_{c, 1}$ and $\vec{C}$ leaves open the Pan-Magellanic hypothesis proposed by Schmidt (1970) and Magalhães et al. (1990), i.e., that the SMC field orientation is an imprint of the geometry of the overall Magellanic system.

To further test this Pan-Magellanic idea, additional RM studies of extragalactic polarized sources behind the Magellanic Bridge will be needed, to see whether the magnetic field in the Bridge potentially also aligns with the vector $\vec{C}$. Meanwhile, the separate possibility that $\vec{B}_{c, 1}$ lies in the SMC disk (which as noted above, lies in a plane only $2^{\circ}$ from the axis defined by the Bridge) provides an important constraint on the origin of the magnetic field in the SMC, as we will discuss fully in $\S 6$ below. We stress that the above analysis is based on the assumption that the RMs and optical starlight polarization probe the same large-scale field in the SMC.

\section{DISCUSSION}

Our observations of the SMC demonstrate the existence of a large-scale coherent magnetic field. A coherent field cannot be explained by compression or stretching of a preexisting random field. The large scale dynamo is the usual mechanism invoked to produce a coherent magnetic field on galactic scales (Beck 2000). In this section, we explore which dynamo (or other) mechanisms might be responsible for producing this coherent field.

\subsection{Ram Pressure Effects}

When galaxies with large scale magnetic fields move rapidly through the intra-cluster medium (ICM), the field lines can be compressed, increasing the total magnetic energy of the system without dynamo action(Otmianowska-Mazur \& Vollmer 2003). Therefore, it is reasonable to consider ram 
pressure as a mechanism that amplifies galactic magnetic fields. The maximum ram pressure considered in the 3D MHD model of Otmianowska-Mazur \& Vollmer (2003) corresponds to a galaxy moving at a velocity of $1500 \mathrm{~km} \mathrm{~s}^{-1}$ through an ICM of density $2 \times 10^{-3} \mathrm{~cm}^{-3}$. The total magnetic energy is increased by a factor of $\sim 5$ in their optimal model during the ram pressure event. Simulated polarized intensity maps show characteristic features during different interaction phases with the ICM. Bright ridges are seen in the compressed region during the compression/stripping phase, while a large scale "ring" field, resembling the field created by a dynamo mechanism, is seen during the gas re-accretion phase in the polarized intensity maps. No such features can be seen in single dish continuum data of the SMC (Loiseau et al. 1987; Haynes et al. 1991). Furthermore, the space velocity of the galaxy used in the model of Otmianowska-Mazur \& Vollmer (2003) is approximately three times larger than that of the velocity of the SMC with respect to the Galactic center (Kallivayalil et al. 2006), while the density of the Milky Way halo is $\sim 10^{-5}$ to $10^{-4} \mathrm{~cm}^{-3}$ at the distance of the SMC (Stanimirović et al. 2002; Sembach 2006). Therefore, the ram pressure effect on the SMC would be roughly 2 orders of magnitude weaker than for the simulations of Otmianowska-Mazur $\&$ Vollmer (2003). Also, it is unclear how ram pressure effects could generate a coherent large scale field from an initial field which might be incoherent. Therefore, we rule out the possibility of ram pressure effects generating the field in the SMC.

\subsection{The Mean-Field Dynamo}

The $\alpha-\omega$ or mean-field dynamo requires turbulence to rise above or below the galactic disk to transform an azimuthal field into a poloidal one (Beck et al. 1996). The radial component of the poloidal field is then transformed back into an azimuthal component by differential rotation of the disk. Although conservation of magnetic helicity can strongly suppress the $\alpha$ effect, it has been shown that this constraint on the mean field dynamo can be alleviated by flows between the disk and the halo, or by galactic outflows, which in turn allow the mean magnetic field to grow to a strength comparable to the equipartition value (see for example Vishniac 2004; Shukurov et al. 2006).

Dynamo action can be characterized by two parameters: $R_{\alpha}$ and $R_{\omega}$, given by Ruzmaikin et al. (1988)

$$
\begin{gathered}
R_{\alpha}=\frac{3 l_{o} \Omega}{u_{0}} \\
R_{\omega}=\frac{3 s \frac{\partial \Omega}{\partial s} h_{0}^{2}}{l_{o} u_{0}}
\end{gathered}
$$

where $l_{o}$ is the outer scale of the turbulence, $s$ is the radial distance from the center of the galaxy, $h_{0}$ is the scale height of the gas disk and $\Omega$ is the angular velocity of the rotating disk. The typical speed, $u_{0}$, of turbulent motion of gas in the SMC can then be approximated by the velocity dispersion in $\mathrm{HI}, u_{0}$ $=22 \pm 2 \mathrm{~km} \mathrm{~s}^{-1}$ (Stanimirović et al. 2004). It is generally believed that supernovae and superbubbles are the main drivers of turbulence in the Galactic disk (McCray \& Snow 1979), so $l_{o}$ is approximately the size of a supernova remnant or a superbubble. We assume that the ISM in the Milky Way, SMC and the LMC have comparable outer scales of turbulence, $l_{o}$ $\sim 90 \mathrm{pc}$ (Gaensler et al. 2005) and gas disk scale heights $h_{0} \sim$ 500 pc (see for example Shukurov 2007). We use the SMC's HI rotation curve obtained by Stanimirović et al. (2004) to characterize its degree of differential rotation. Under the condition $^{6}$ that $\mathrm{R}_{\omega} \gg \mathrm{R}_{\alpha}$, we can compute the dynamo number, $D$, a dimensionless parameter which determines the growth rate of the magnetic field (Ruzmaikin et al. 1988):

$$
D=R_{\alpha} R_{\omega}=\frac{9 \Omega s h_{0}^{2}}{u_{0}^{2}} \frac{\partial \Omega}{\partial s} .
$$

Note that the above equation is independent of the turbulent outer scale. Dynamo numbers at radii ranging from $s=0.5$ to $3.2 \mathrm{kpc}$ are computed. In this range, the amount of shear in the disk of the SMC is given by $s \frac{\partial \Omega}{\partial s} \sim 10^{-16} \mathrm{~s}^{-1}$, which is comparable to the shear in the Galactic disk near the sun ${ }^{7}$ $\sim 5 \times 10^{-16} \mathrm{~s}^{-1}$. We obtain values of $|\mathrm{D}|$ ranging from 0 to 4 in the SMC, while for the Milky Way, $|\mathrm{D}| \sim 20$ in the solar vincinity (Shukurov 2007). The critical value for an exponential growth of the field is given by $\left|D_{\text {critical }}\right| \sim 8-10$, while a sub-critical dynamo number implies no growth (Shukurov 2007). We thus conclude that for the SMC, the classical mean field dynamo is not at work.

Using statistical studies of the SMC's neutral hydrogen, Stanimirović \& Lazarian (2001) found no characteristic scale of turbulence up to the size of the galaxy. This implies that the turbulent outer scale $l_{o}$ could be up to a few kpcs and the value $\mathrm{R}_{\omega}$ would then be much smaller than $\mathrm{R}_{\alpha}$. In this case, the dynamo number obtained using Equation (38) is no longer a good description of the field growth rate, since both the $\alpha-\omega$ and $\alpha^{2}$ dynamos (the latter is a dynamo driven by helical turbulence action alone) will operate. In this case the dynamo number $|\mathrm{D}|$ would increase by $\sim 30 \%$ (Ruzmaikin et al. 1988), which is not enough to rise the dynamo number above the critical level. Moreover, since the SMC experienced bursts of star formation triggered by tidal interactions $\sim 0.4$ and 2.5 Gyrs ago (Zaritsky \& Harris 2004), the additional energy injected into the ISM could have created outflows that would constantly disrupt the buildup of a large scale magnetic field produced by the $\alpha-\omega$ dynamo. We draw the conclusion that the mean-field dynamo is likely not responsible for the observed coherent field in the SMC.

\subsection{The Fluctuating Dynamo}

It is thought that when the large scale dynamo is ineffective, as may occur in weakly rotating galaxies such as the SMC, the fluctuating dynamo (or the small-scale dynamo) can become important. The fluctuating dynamo, unlike the largescale dynamo, can work without differential rotation in the galactic disk and can generate magnetic field with a correlation length similar to the energy carrying scale of the turbulence (Shukurov 2007). The fluctuating dynamo is believed to operate in small and slowly rotating galaxies with enhanced star formation, such as IC 10 (Chyży et al. 2003). The typical field amplification time scale is $10^{6}$ to $10^{7}$ years, much shorter than the standard dynamo growth rate. Signatures of random magnetic fields created by a fluctuating dynamo are isolated polarized non-themal regions coinciding with locations of star formation (Chyży et al. 2003). Since magnetic fields produced by a fluctuating dynamo are incoherent on galactic scales, they cannot be responsible for producing the observed coherent field in the SMC. However, the random field strength

\footnotetext{
${ }^{6}$ For an energy injection scale of value $l_{o} \sim 90 \mathrm{pc}$, the condition that $\mathrm{R}_{\omega}$ $\gg \mathrm{R}_{\alpha}$ is satisfied.

${ }^{7}$ Adopting a value for Oort's constant $\mathrm{A} \sim 15 \mathrm{~km} \mathrm{~s}^{-1} \mathrm{kpc}^{-1}$
} 
( $\sim 3 \mu \mathrm{G})$ estimated in the SMC in $\S 4.3$ suggests that the fluctuating dynamo could be responsible for producing the random field component. Single dish radio continuum data of the SMC at multiple wavelengths show global diffuse synchrotron emission (Loiseau et al. 1987; Haynes et al. 1991), which also suggests that the random field strength in the SMC might be relatively high.

\subsection{The Cosmic-Ray Driven Dynamo}

Parker (1992) proposed a cosmic-ray driven dynamo that has a much shorter amplification time scale than the standard mean-field dynamo. In this model, the driving force comes from cosmic rays injected into the galactic disk from the acceleration of charged particles in SNR shocks. Unlike the standard dynamo, this model incorporates a set of interacting forces including the buoyancy of cosmic rays, the Coriolis force, differential rotation and magnetic reconnection (Hanasz \& Lesch 1998). Differential rotation of the galactic disk is still required but the considerably larger $\alpha$ effect allows weakly rotating galaxies to achieve a supercritical dynamo number. The first numerical magneto-hydrodynamic (MHD) model of the CR-driven dynamo was developed by Hanasz \& Lesch (2004). They modeled a differentially rotating galaxy with a constant supply of cosmic rays and found that the largescale magnetic field amplification time scale was about 250 Myrs. OB associations and frequent supernova explosions during the bursts of star formation in the SMC could result in a large cosmic ray flux, allowing the amplification of magnetic field in the SMC via the Parker-type dynamo. If the fast dynamo is responsible for the observed field due to the tidal triggered star formation episode $\sim 0.2$ Gyrs ago, it would have just enough time to build up a galactic scale field before the tidal velocity field damps the dynamo effect (Kronberg 1994; Chyży \& Beck 2004). This can potentially also explain the coherent spiral field seen in the LMC (Gaensler et al. 2005).

Otmianowska-Mazur et al. (2000) modeled the magnetic field in the LMC-type irregular galaxy NGC 4449 using a value of $R_{\alpha}$ comparable to that of a fast dynamo. NGC 4449 is found to display "fan" like structures that mimic magnetic spiral arms in polarized intensity. The Faraday rotation map of NGC 4449 suggests that the galaxy hosts a coherent field (Klein et al. 1996; Chyży et al. 2000). Otmianowska-Mazur et al. (2000) consider a model galaxy with a radius of $\sim 2.5$ $\mathrm{kpc}$ and a maximum rotational velocity of about $30 \mathrm{~km} \mathrm{~s}^{-1}$, which is similar to the SMC. No outflow from a bar and no random field were included. The value for $l_{0}^{2} \Omega / h_{0}$ was $5 \mathrm{~km}$ $\mathrm{s}^{-1}$ and the turbulent diffusivity $\left(\eta \sim l_{0} u_{0} / 3\right)$ was chosen to be $1.5 \times 10^{26} \mathrm{~cm}^{2} \mathrm{~s}^{-1}$. These parameters are typical of a cosmic ray dynamo as shown by Hanasz et al. (2004). Evolving the modeled galaxy using the above parameters over $\sim 0.1 \mathrm{Gyr}$ leads to an increase in the total magnetic energy. Also, this model is able to reproduce spiral-like field structure resembling the observation of NGC 4449. However, it does not include several possibly important physical processes. First, the SMC is likely to be subjected to an injection of random field into the ISM due to a fluctuating dynamo (see $\S 6.3$ ), which this model does not account for. Second, the SMC has a rotation curve which peaks at $\sim 50 \mathrm{~km} \mathrm{~s}^{-1}$ rather than the $30 \mathrm{~km}$ $\mathrm{s}^{-1}$ used by Otmianowska-Mazur et al. (2000). This results in a more effective $\omega$ effect, which increases the growth rate of the magnetic field, while random field injection increases the total magnetic energy of the galaxy faster. No simulated Faraday rotation map was produced by Otmianowska-Mazur et al.
(2000), therefore, no direct comparison can be made between their model and our data. MHD models devoted to simulate the growth of the magnetic field in the SMC are needed in order to provide a definitive conclusion.

We have established above that it is possible for the cosmicray driven dynamo to produce the observed magnetic field in the SMC in terms of time scale arguments. Let us now consider whether this dynamo can explain the observed field geometry. In $\S 5$, we showed that the 3D magnetic field vector of the SMC may lie in the disk of the galaxy and that it may align with the vector joining the Magellanic Clouds. A dynamo produces an azimuthal magnetic field that predominantly lies in the disk of a galaxy (see Ruzmaikin et al. 1988), and this could account for the potential alignment of the SMC's magnetic field with the SMC disk as calculated in $\S 5$. If the field is aligned with the Bridge rather than the disk (as also allowed by the range of angles calculated in $\S 5$ ), this could be understood as resulting from ongoing tidal interactions between the Magellanic Clouds, which could provide a slight realignment of the overall field orientation.

If the cosmic-ray driven dynamo is the underlying mechanism that produces the magnetic field in the SMC, it also needs to explain the unidirectional field lines seen across the galaxy. The magnetic field configuration in a galaxy can be decomposed into different dynamo modes (Beck et al. 1996). The strongest dynamo mode in an axisymmetric disk is the $m=0$ mode, followed by a weaker bisymmetric $(m=1)$ mode. It has been suggested by Moss (1995) that tidal interactions can generate bisymmetric magnetic fields in galaxies, provided that the axisymmetric mode is already at work. Observations show that in interacting galaxies, such as M51 and M81, the bisymmetric mode can be important (Krause et al. 1989). According to the 3D mean field dynamo model studied by Vögler \& Schmitt (2001), non-axisymmetric gas motion is induced in galactic disks during tidal interaction, and can damp the usual dominant $m=0$ mode and excite the $m=1$ mode when the induced tidal velocity is small. An axisymmetric magnetic field would exhibit a change in the sign of RM across the disk of the galaxy when viewed edge on whereas a bisymmetric magnetic field would vary doubleperiodically with the azimuthal angle (Krause et al. 1989). It is unclear how the superposition of a $m=0$ mode and a $m=1$ mode could produce unidirectional field lines with negative $\mathrm{RM}$ across the SMC, because a superposition of higher order modes will result in more RM sign changes across the galaxy disk when viewed edge on.

The observed unidirectional magnetic field lines and the possible alignment of the field with the Magellanic Bridge could be explained as follows. Cosmic ray driven dynamo produces a predominately azimuthal magnetic field in the SMC disk; this field is then stretched tidally along the SMCLMC axis, maintaining its orientation when projected onto the plane of the sky (to produce starlight polarization vectors of similar orientation). Note that RM is non-zero only when the average line of sight electron density is non-zero. It is possible that the field lines in the SMC do close, that is, there are sight lines along which field lines do point towards us, but only at locations with low EM off the main body of the SMC. Only half of the displaced magnetic loop, whose line of sight component is directed away from us, would then be observed. The other half of the loop whose line of sight component is directed towards us would not show positive RMs, as it should coincide with regions of low EM .

To summarize, the cosmic-ray driven dynamo is a possible 
field generation mechanism for the SMC but has difficulties explaining the observed magnetic field geometry. One has to explain the fact that the observed field is unidirectional and that it potentially lies in the disk of the SMC and aligns with the Magellanic Bridge. Current observational data are not sufficient to rule out/prove the cosmic-ray driven dynamo; further observational tests are needed.

\section{CONCLUSIONS}

We have measured the Faraday rotation of extragalactic polarized sources behind the Small Magellanic Cloud to determine the SMC's magnetic field strength and geometry. Our study reveals that the SMC has a galactic-scale field of 0.19 $\pm 0.06 \mu \mathrm{G}$ directed coherently away from us along the line of sight. Optical polarization data on stars in the SMC are reanalyzed using the Chandradsekhar-Fermi method to give an ordered component of the magnetic field in the plane of the sky, of strength $1.6 \pm 0.4 \mu \mathrm{G}$. Under the assumption that the Faraday rotation measures and optical starlight polarization probe the same underlying large scale field in the SMC, we have constructed a $3 \mathrm{D}$ magnetic field vector of the SMC. It is found that this magnetic field vector possibly aligns with the Magellanic Bridge. This potential alignment needs to be verified by future studies of RMs towards extragalactic sources behind the Magellanic Bridge. The random magnetic field strength in the SMC derived from RM data alone and that derived by combining the results of the $\mathrm{C}-\mathrm{F}$ method with equipartition were found to be in agreement $(\sim 3 \mu \mathrm{G})$. This implies that our underlying assumption, that these 3 independent methods probe different components of the same large scale field, is self-consistent.

The SMC is a slowly rotating galaxy, for which the standard mean-field dynamo is not expected to be at work be- cause of the subcritical dynamo number. The cosmic-ray driven dynamo has a short enough amplification time scale to explain the observed coherent field. With modifications by tidal interactions, the field generated by the cosmic-ray driven dynamo could potentially be aligned with the Magellanic Bridge. However, this model faces difficulties in explaining the observed uni-directional field lines. Therefore, the origin of the magnetic field in the SMC is still an open question which needs to be followed up with more observations. The relatively small number of background rotation measures makes it difficult to interpret the observed RMs in detail. Future observations of the SMC with the Square Kilometre Array will provide $\sim 10^{5} \mathrm{RMs}$ in a field of 40 square degrees surrounding the SMC (Beck \& Gaensler 2004); with which different possible origins of the magnetic field in the SMC can be fully evaluated (Stepanov et al. 2008).

Acknowledgements We thank Joseph Gelfand for carrying out the ATCA observations, Anvar Shukurov, Erik Muller, Alyssa Goodman and Douglas Finkbeiner for useful discussions, and Rainer Beck, Marita Krause and Ellie Berkhuijsen for their help and hospitality during S. A. M. 's visit to the Max Planck Institute for Radio Astronomy. M. H. acknowledges support from the National Radio Astronomy Observatory (NRAO), which is operated by Associated Universities Inc., under cooperative agreement with the National Science Foundation. This research was supported by the National Science Foundation through grant AST-0307358 to Harvard College Observatory. The Australia Telescope Compact Array is part of the Australian Telescope, which is funded by the Commonwealth of Australia for operation as a National Facility managed by CSIRO. The Southern H-Alpha Sky Survey Atlas (SHASSA) is supported by the NSF.

Facilities: ATCA

\section{APPENDIX}

\section{A. EXTINCTION CORRECTION OF H $\alpha$ EMISSION}

We here describe the procedure to derive the intrinsic H $\alpha$ intensity of the SMC. We assume that dust is well mixed with H $\alpha$ emitting gas for both the SMC and the Milky Way. The observed $\mathrm{H} \alpha$ emission is given by

$$
I_{\text {observed }}=\frac{I_{\text {instrinic }, \mathrm{SMC}}}{\tau_{\mathrm{H} \alpha, \mathrm{SMC}}}\left(1-e^{\tau_{\mathrm{H} \alpha, \mathrm{SMC}}}\right) e^{-\tau_{\mathrm{H} \alpha, \mathrm{MW}}}+\frac{I_{\text {intrinsic }, \mathrm{MW}}}{\tau_{\mathrm{H} \alpha, \mathrm{MW}}}\left(1-e^{-\tau_{\mathrm{H} \alpha, \mathrm{MW}}}\right)
$$

where $\tau_{\mathrm{H} \alpha \text {,SMC }}$ is the optical depth of $\mathrm{H} \alpha$ in the SMC, $\tau_{\mathrm{H} \alpha, \mathrm{MW}}$ is the optical depth of $\mathrm{H} \alpha$ in the Milky Way, $I_{\text {intrinsic,Mw }}$ is the intrinsic $\mathrm{H} \alpha$ emission of the Milky Way, and $I_{\text {intrinsic,SMC }}$ is the intrinsic $\mathrm{H} \alpha$ emission of the SMC. The second term in the above equation can be estimated by the observed off source $\mathrm{H} \alpha$ intensity in the regions surrounding the SMC.

The uncertainty in estimating the intrinsic $\mathrm{H} \alpha$ intensity mainly results from the location of the dust with respect to the $\mathrm{H} \alpha$

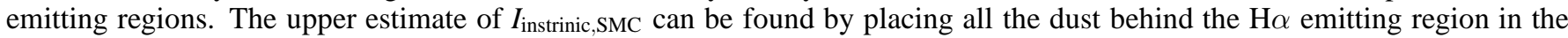
$\mathrm{SMC}$, so that what we observe is the intrinsic $\mathrm{H} \alpha$ intensity extincted only by the foreground Milky Way dust. The lower estimate of $I_{\text {instrinic,SMC }}$ can be found by placing all the dust in front of the $\mathrm{H} \alpha$ emitting region in the SMC.

\section{B. A MODEL TO ESTIMATE THE RANDOM MAGNETIC FIELD STRENGTH}

We construct this model based on Gaensler et al. $(2001,2005)$ and ionized gas model 2 (see $\S 3.3 .2$ ), for which case we assume that the average electron density $\left(\overline{n_{e}}\right)$ along different lines of sight is the same but the depth of the SMC varies from one sight line to the other. From model 3, the mean depth through the SMC is $\langle L\rangle \approx 10 \mathrm{kpc}$ with a standard deviation $\Delta L \approx 6 \mathrm{kpc}$. Suppose that the depth of the SMC through a particular sight line is $L$, divided up into cells of linear size $l_{o}$. The total number of cells one looks through along the line of sight is given by

$$
N=\frac{L}{l_{o}}
$$

Within each cell, we suppose that the magnetic field is composed of a coherent component of strength $B_{c}$ (same direction and strength from cell to cell), whose strength along the line of sight is $\left\langle B_{c, \|}\right\rangle \approx 0.16 \mu \mathrm{G}$, and a random component of strength $B_{r}$ oriented at an angle $\theta_{\text {cell,i }}$ with respect to the line of sight. The component of the random field along the line of sight is

$$
B_{r, \|}=B_{r} \cos \theta_{\text {cell }, \mathrm{i}}
$$


The line of sight magnetic field strength in a cell is given by

$$
B_{\|}=\left\langle B_{c, \|}\right\rangle+B_{r, \|}=\left\langle B_{c, \|}\right\rangle+B_{r} \cos \theta_{\text {cell }, \mathrm{i}}
$$

In addition, we assume that the random component is coherent within each cell but that $\theta$ varies randomly from cell to cell. Different levels of Faraday rotation will be experienced by the incident light rays because they pass through different series of cells and different numbers of cells. Linearly polarized light which passes through a single cell in the SMC experiences a Faraday rotation given by

$$
\mathrm{RM}_{1-\text { cell }}=0.812 n_{e_{\text {cell }, i}} l_{o} \overline{B_{\|}}=0.812 n_{e_{\text {cell }, i}} l_{o}\left(\left\langle B_{c, \|}\right\rangle+B_{r} \cos \theta_{\text {cell }, \mathrm{i}}\right)
$$

After passing through $N$ cells, the incident radiation experiences a Faraday rotation of

$$
\mathrm{RM}_{N-\text { cells }}=0.812 l_{o} B_{r} \sum_{i=1}^{N} n_{e_{\text {cell }, i}} \cos \theta_{\text {cell }, \mathrm{i}}+0.812 l_{o}\left\langle B_{c, \|}\right\rangle \sum_{i=1}^{N} n_{e_{\text {cell, }, \mathrm{i}}}
$$

where

$$
\sum_{i=1}^{N} n_{e_{\mathrm{cell}, \mathrm{i}}}=\overline{n_{e}} N
$$

Since the electron density does not correlate with the orientation of the random field in individual cells,

$$
\sum_{i=1}^{N} n_{e_{\text {cell }, \mathrm{i}}} \cos \theta_{\mathrm{cell}, \mathrm{i}}=\overline{n_{e}} \sum_{i=1}^{N} \cos \theta_{\mathrm{cell}, \mathrm{i}} .
$$

One can rewrite the expression for the rotation measure of the radiation after passing through $\mathrm{N}$ cells as

$$
\mathrm{RM}_{\mathrm{N}-\mathrm{cells}}=0.812 l_{o} B_{r} \bar{n}_{e} \sum_{i=1}^{N} \cos \theta_{\text {cell, } \mathrm{i}}+0.812 l_{o}\left\langle B_{c, \|}\right\rangle N \overline{n_{e}}
$$

Averaging across different sight lines, the mean RM through the SMC is given by

$$
\langle\mathrm{RM}\rangle=0.812 l_{o}\left\langle B_{c, \|}\right\rangle\langle N\rangle
$$

where $\langle N\rangle=\langle L\rangle / l_{o}$ is the average number of cells along different sight lines.

Using the central limit theorem for large $N$, the standard deviation of RM through the SMC can be expressed as

$$
\sigma_{\mathrm{RM}}=0.812 l_{o} \overline{n_{e}} \sqrt{\left\langle B_{c, \|}\right\rangle^{2}\left(\frac{\Delta L}{l_{o}}\right)^{2}+B_{r}{ }^{2}\left(\frac{\langle L\rangle}{3 l_{o}}\right)} .
$$




\section{REFERENCES}

Beck, R. 1982, A\&A, 106, 121

Beck, R. 2000, Philos. Trans. Roy. Soc. London A., 358, 777

Beck, R. 2004, Ap\&SS, 289, 293

Beck, R. 2007, in EAS Publications Series, Vol. 23, Sky polarisation at farinfrared to radio wavelengths (Les Ulis, France: EDP Sciences), 19-36

Beck, R., Brandenburg, A., Moss, D., Shukurov, A., \& Sokoloff, D. 1996, ARA\&A, 34, 155

Beck, R. \& Gaensler, B. M. 2004, New Ast. Rev., 48, 1289

Beck, R. \& Krause, M. 2005, Astro. Nach., 326, 414

Beck, R., Shukurov, A., Sokoloff, D., \& Wielebinski, R. 2003, A\&A, 411, 99

Bekki, K. \& Chiba, M. 2005, MNRAS, 356, 680

Berkhuijsen, E. M., Mitra, D., \& Mueller, P. 2006, Astro. Nach., 327, 82

Besla, G., Kallivayalil, N., Hernquist, L., Robertson, B., Cox, T. J., van der Marel, R. P., \& Alcock, C. 2007, ApJ, 668, 949

Bevington, P. R. \& Robinson, D. K. 2003, Data Reduction and Error Analysis for the Physical Sciences (New York: McGraw-Hill)

Broten, N. W., MacLeod, J. M., \& Vallee, J. P. 1988, Ap\&SS, 141, 303

Brown, J. C., Taylor, A. R., \& Jackel, B. J. 2003, ApJS, 145, 213

Chandrasekhar, S. \& Fermi, E. 1953, ApJ, 118, 113

Chi, X. \& Wolfendale, A. W. 1993, Nature, 362, 610

Chyży, K. T. \& Beck, R. 2004, A\&A, 417, 541

Chyży, K. T., Beck, R., Kohle, S., Klein, U., \& Urbanik, M. 2000, A\&A, 355, 128

Chyży, K. T., Knapik, J., Bomans, D. J., Klein, U., Beck, R., Soida, M., \& Urbanik, M. 2003, A\&A, 405, 513

Cioni, M.-R. L., van der Marel, R. P., Loup, C., \& Habing, H. J. 2000, A\&A, 359,601

Cochran, W. G. 1977, Sampling Techniques (3rd Edn) (New York: Wiley)

Cordes, J. M. \& Lazio, T. J. W. 2002, astro-ph/0207156

Crawford, F., Manchester, R. N., \& Kaspi, V. M. 2001, AJ, 122, 2001

Crutcher, R., Heiles, C., \& Troland, T. 2003, in Turbulence and Magnetic Fields in Astrophysics, ed. E. Falgarone \& T. Passot (Berlin: Springer), $155-181$

Dufour, R. J. \& Harlow, W. V. 1977, ApJ, 216, 706

Filipović, M. D., Payne, J. L., Reid, W., Danforth, C. W., Staveley-Smith, L., Jones, P. A., \& White, G. L. 2005, MNRAS, 364, 217

Gaensler, B. M., Dickey, J. M., McClure-Griffiths, N. M., Green, A. J., Wieringa, M. H., \& Haynes, R. F. 2001, ApJ, 549, 959

Gaensler, B. M., Haverkorn, M., Staveley-Smith, L., Dickey, J. M., McClureGriffiths, N. M., Dickel, J. R., \& Wolleben, M. 2005, Science, 307, 1610

Gardiner, L. T., Sawa, T., \& Fujimoto, M. 1994, MNRAS, 266, 567

Gaustad, J. E., McCullough, P. R., Rosing, W., \& Van Buren, D. 2001, PASP, 113,1326

Hanasz, M., Kowal, G., Otmianowska-Mazur, K., \& Lesch, H. 2004, ApJ, 605, L33

Hanasz, M. \& Lesch, H. 1998, A\&A, 332, 77

Hatzidimitriou, D., Croke, B. F., Morgan, D. H., \& Cannon, R. D. 1997, A\&AS, 122, 507

Haynes, R. F., Harnett, S. W. J. I., Klein, U., Wielebinski, R., Buczilowski, U. R., Bajaja, E., Malin, D., Murray, J. D., et al. 1990, in IAU Symposium, Vol. 140, Galactic and Intergalactic Magnetic Fields, ed. R. Beck, R. Wielebinski, \& P. P. Kronberg (Dordrecht, Netherlands: Kluwer Academic Publishers), 205

Haynes, R. F., Klein, U., Wayte, S. R., Wielebinski, R., Murray, J. D., Bajaja, E., Meinert, D., Buczilowski, U. R., et al. 1991, A\&A, 252, 475

Haynes, R. F., Murray, J. D., Klein, U., \& Wielebinski, R. 1986, A\&A, 159, 22

Heitsch, F., Zweibel, E. G., Mac Low, M.-M., Li, P., \& Norman, M. L. 2001, ApJ, 561, 800

Henize, K. G. 1956, ApJS, 2, 315

Hopkins, A. M., Miller, C. J., Connolly, A. J., Genovese, C., Nichol, R. C., \& Wasserman, L. 2002, AJ, 123, 1086

Johnston-Hollitt, M., Hollitt, C. P., \& Ekers, R. D. 2004, in The Magnetized Interstellar Medium, ed. B. Uyaniker, W. Reich, \& R. Wielebinski (Katlenburg-Lindau, Germany: Copernicus GmbH), 13-18

Jones, A. P., Tielens, A. G. G. M., \& Hollenbach, D. J. 1996, ApJ, 469, 740

Kallivayalii, N., van der Marel, R. P., \& Alcock, C. 2006, ApJ, 652, 1213

Klein, U., Hummel, E., Bomans, D. J., \& Hopp, U. 1996, A\&A, 313, 396

Krause, M., Beck, R., \& Hummel, E. 1989, A\&A, 217, 17

Kronberg, P. P. 1994, Rep. Prog. Phys., 217, 58

Lagache, G., Abergel, A., Boulanger, F., Désert, F. X., \& Puget, J.-L. 1999, A\&A, 344, 322

Lah, P., Kiss, L. L., \& Bedding, T. R. 2005, MNRAS, 359, L42

Lequeux, J. 2005, The interstellar medium (Berlin: Springer)

Lockman, F. J. \& Condon, J. J. 2005, AJ, 129, 1968
Loiseau, N., Klein, U., Greybe, A., Wielebinski, R., \& Haynes, R. F. 1987, A\&A, 178, 62

Madsen, G. J., Reynolds, R. J., \& Haffner, L. M. 2006, ApJ, 652, 401

Magalhães, A. M., Loiseau, N., Rodrigues, C. V., \& Piirola, V. 1990, in IAU Symposium, Vol. 140, Galactic and Intergalactic Magnetic Fields, ed. R. Beck, R. Wielebinski, \& P. P. Kronberg (Dordrecht, Netherlands: Kluwer Academic Publisher), 255

Manchester, R. N., Fan, G., Lyne, A. G., Kaspi, V. M., \& Crawford, F. 2006, ApJ, 649, 235

Manchester, R. N., Hobbs, G. B., Teoh, A., \& Hobbs, M. 2005, AJ, 129, 1993

Mathewson, D. S. \& Ford, V. L. 1970a, AJ, 75, 778

Mathewson, D. S. \& Ford, V. L. 1970b, ApJ, 160, L43

McCray, R. \& Snow, Jr., T. P. 1979, ARA\&A, 17, 213

Melrose, D. B. 1980, Plasma Astrophysics. Nonthermal processes in diffuse magnetized plasmas - Vol.2: Astrophysical applications (New York: Gordon and Breach)

Moss, D. 1995, MNRAS, 275, 191

Myers, P. C. \& Goodman, A. A. 1991, ApJ, 373, 509

Otmianowska-Mazur, K., Chyży, K. T., Soida, M., \& von Linden, S. 2000, A\&A, 359, 29

Otmianowska-Mazur, K. \& Vollmer, B. 2003, A\&A, 402, 879

Pacholczyk, A. G. 1970, Radio astrophysics. Nonthermal processes in galactic and extragalactic sources (San Francisco: Freeman)

Parker, E. N. 1992, ApJ, 401, 137

Pei, Y. C. 1992, ApJ, 395, 130

Peimbert, M. \& Peimbert, A. 2000, in The Light Elements and their Evolution, ed. L. da Silva, R. de Medeiros, \& M. Spite, IAU Symposium 198 (San Francisco: ASP), 194

Piatek, S., Pryor, C., \& Olszewski, E. W. 2008, arXiv:0712.1764, Accepted for publication in $\mathrm{AJ}$

Pohl, M. 1993, A\&A, 279, L17

Reynolds, R. J. 1991, ApJ, 372, L17

Rodrigues, C. V., Magalhaes, A. M., Coyne, G. V., \& Piirola, V. 1997, ApJ, 485,618

Russell, S. C. \& Dopita, M. A. 1992, ApJ, 384, 508

Ruzmaikin, A. A., Sokolov, D. D., \& Shukurov, A. M. 1988, Magnetic Fields of Galaxies (Dordrecht: Kluwer)

Sandstrom, K. M. 2001, Senior Undergraduate Thesis (Cambridge: Harvard University)

Sanduleak, N. 1968, AJ, 73, 246

Sanduleak, N. 1969, AJ, 74, 877

Sanduleak, N. \& Philip, A. G. D. 1968, AJ, 73, 566

Sault, R. J. \& Killeen, N. E. B. 2003, The Miriad User's Guide (Sydney: ATNF)

Schmidt, T. 1970, A\&A, 6, 294

Schmidt, T. 1976, A\&AS, 24, 357

Schwering, P. B. W. \& Israel, F. P. 1991, A\&A, 246, 231

Sembach, K. R. 2006, in The Local Group as an Astrophysical Laboratory, ed. M. Livio \& T. M. Brown, Space Telescope Science Institute symposium series (Cambridge, UK: Cambridge University Press), 86-99

Shukurov, A., Sokoloff, D., Subramanian, K., \& Brandenburg, A., 2006, A\&A, 448, L33

Shukurov, A. 2007, in Mathematical Aspects of Natural Dynamos, ed. E. Dormy \& A. M. Soward (Boca Raton: CRC Press), 313-358

Stanimirović, S., Dickey, J. M., Krčo, M., \& Brooks, A. M. 2002, ApJ, 576, 773

Stanimirović, S. \& Lazarian, A. 2001, ApJ, 551, L53

Stanimirović, S., Staveley-Smith, L., Dickey, J. M., Sault, R. J., \& Snowden, S. L. 1999, MNRAS, 302, 417

Stanimirović, S., Staveley-Smith, L., \& Jones, P. A. 2004, ApJ, 604, 176

Stanimirović, S., Staveley-Smith, L., van der Hulst, J. M., Bontekoe, T. R., Kester, D. J. M., \& Jones, P. A. 2000, MNRAS, 315, 791

Stepanov, R., Arshakian, T. G., Beck, R., Frick, P., \& Krause, M. 2008, arXiv:0711.1267, Accepted for publication in A\&A

Tinbergen, J. 1996, Astronomical Polarimetry (Cambridge, UK: Cambridge University Press), 174

Vishniac, E. 2004, Proceedings of The Magnetized Plasma in Galaxy Evolution, eds. K. T. Chyży, K. Otmianowska-Mazur, M. Soida \& R.-J. Dettmar (Kraków, Poland: Jagiellonian University)

Vögler, A. \& Schmitt, D. 2001, A\&A, 374, 36

Wayte, S. R. 1990, ApJ, 355, 473

Westerlund, B. E. 1990, A\&A Rev., 2, 29

Widrow, L. M. 2002, Rev. Mod Phys, 74, 775

Yoshizawa, A. M. \& Noguchi, M. 2003, MNRAS, 339, 1135

Zaritsky, D. \& Harris, J. 2004, ApJ, 604, 167 
TABLE 1

A LIST OF SYMBOLS USED IN THIS PAPER

\begin{tabular}{|c|c|c|}
\hline Symbol & Physical Quantity & Section in which first used \\
\hline$\alpha$ & spectral index of synchrotron emission defined by $I_{\nu} \propto \nu^{-\alpha}$ & $\S 1.2$ \\
\hline a & angular offset in degrees eastward from $\mathrm{RA}=0$ & $\S 2.1$ \\
\hline$B_{\|}(l)$ & magnetic field as a function of path length $l$ along the line of sight in units of $\mu \mathrm{G}$ & $\S 1.3$ \\
\hline$\frac{11}{B_{\|}}$ & average magnetic field strength along the line of sight in $\mu \mathrm{G}$ & $\$ 3.3$ \\
\hline$\left\langle B_{o, \perp}\right\rangle$ & average magnetic field strength in the plane of the sky in units of $\mu \mathrm{G}$ & $\S 1.4$ \\
\hline$B_{\|, i}$ & individual measurement of line-of-sight magnetic field $\overline{B_{\|}}$through the SMC in units of $\mu \mathrm{G}$ & $\S 3.3 .4$ \\
\hline$\left\langle B_{c, \perp}\right\rangle$ & strength of the coherent component of the magnetic field in the plane of the sky in units of $\mu \mathrm{G}$ & $\S 4.1$ \\
\hline$\left\langle B_{\text {total }, \perp\rangle}\right\rangle$ & strength of the total equipartition magnetic field in the plane of the sky in units of $\mu \mathrm{G}$ & $\$ 4.2$ \\
\hline$B_{r}$ & strength of random magnetic field in units of $\mu \mathrm{G}$ & $\$ 4.3$ \\
\hline$\sigma_{B, i}$ & uncertainty associated with an individual magnetic field measurement $B_{\|, i}$ in units of $\mu \mathrm{G}$ & $\$ 3.3 .4$ \\
\hline$B_{r, \|}$ & strength of random magnetic field parallel to the line of sight in units of $\mu \mathrm{G}$ & $\S \mathrm{B}$ \\
\hline$\left\langle B_{c, \|}\right\rangle$ & coherent magnetic field parallel to the line of sight averaged across the SMC in units of $\mu \mathrm{G}$ & $\$ 3.3 .4$ \\
\hline$\left\langle B_{r, \perp}\right\rangle$ & random magnetic field strength perpendicular to the line of sight in units of $\mu \mathrm{G}$ & $\S 4.3$ \\
\hline$B_{\text {total }, c}$ & total coherent magnetic field strength in units of $\mu \mathrm{G}$ & $\S 5$ \\
\hline$\vec{B}_{c, 1}, \vec{B}_{c, 2}$ & $\begin{array}{l}\text { the 3D magnetic field vector of the SMC, subscripts } 1 \text { and } 2 \text { indicates the two vectors whose } \\
\text { line-of-sight component is the same but have oppositely directed plane-of-the-sky component }\end{array}$ & $\S 5$ \\
\hline$\gamma_{H}$ & equivalent molecular weight of the ISM for SMC abundance & $\S 4.1$ \\
\hline$\gamma_{e}$ & power law index of electron energy distribution in cosmic rays & $\S 4.2$ \\
\hline$\vec{C}$ & the vector joining the center of the LMC to the center of the SMC & $\S 5$ \\
\hline$\Delta \phi$ & angle that the polarization plane rotates through in radians & $\S 1.3$ \\
\hline$\overline{\delta_{p}}$ & angular deviation of $\theta_{p}$ from $\left\langle\theta_{p}\right\rangle$ & $\S 1.4$ \\
\hline$D$ & dynamo number & $\S 6.2$ \\
\hline$D_{\text {critical }}$ & critical dynamo number & $\S 6.2$ \\
\hline $\mathrm{DM}$ & dispersion measure in units of $\mathrm{pc} \mathrm{cm}^{-3}$ & $\S 3.1$ \\
\hline$\left\langle\mathrm{DM}_{\mathrm{SMC}, \mathrm{pulsar}}\right\rangle$ & average dispersion measure towards SMC pulsars, after foreground subtraction & $\S 3.1$ \\
\hline$\left\langle\mathrm{DM}_{\mathrm{SMC}}\right\rangle$ & average dispersion measure through the SMC & $\S 3.1$ \\
\hline$\sigma_{\mathrm{DM}}$ & standard deviation of pulsar DMs in the SMC & $\S 3.1$ \\
\hline EM & emission measure in units of $\mathrm{pc} \mathrm{cm}^{-6}$ & $\$ 3.2$ \\
\hline$\left\langle\mathrm{EM}_{\mathrm{SMC}}\right\rangle$ & average emission measure through the $\mathrm{SMC}$ in $\mathrm{pc} \mathrm{cm}^{-6}$ & $\$ 3.3 .2$ \\
\hline $\mathrm{EM}_{\text {source }}$ & emission measure towards an individual extragalactic source behind the SMC & $\$ 3.3 .2$ \\
\hline$f$ & filling factor of thermal electrons along the line of sight & $\$ 3.3 .2$ \\
\hline$f L$ & occupation length in units of $\mathrm{pc}$ of thermal electrons along the line of sight & $\$ 3.3 .3$ \\
\hline$h_{0}$ & scale height of galactic disk in pc & $\S 6.2$ \\
\hline$\eta$ & turbulent diffusivity in units of $\mathrm{cm}^{2} \mathrm{~s}^{-1}$ & $\S 6.4$ \\
\hline$\theta_{\text {cell }, i}$ & orientation of the random magnetic field with respect to the line of sight in cell $i$ & $\S \mathrm{B}$ \\
\hline$I_{\nu}$ & specific intensity of synchrotron emission in erg s $\mathrm{s}^{-1} \mathrm{~cm}^{-2} \mathrm{~Hz}^{-1} \mathrm{sr}^{-1}$ & $\S 1.2$ \\
\hline$I_{\mathrm{H} \alpha, \text { intrinsic,SMC }}$ & intrinsic $\mathrm{H} \alpha$ intensity of the SMC in units of Rayleighs & $\S 3.2$ \\
\hline$I_{\text {observed }}$ & observed $\mathrm{H} \alpha$ intensity towards the SMC & $\S \mathrm{A}$ \\
\hline$I_{\text {instrinic, SMC }}$ & H $\alpha$ intensity of the SMC after extinction correction for both the Milky Way and the SMC & $\S \mathrm{A}$ \\
\hline$I_{\text {intrinsic.MW }}$ & $\mathrm{H} \alpha$ intensity of the Milky Way after extinction correction & $\S \mathrm{A}$ \\
\hline & dimensionless galactic dust-to-gas ratio defined in Equation 9 & $\S 3.2$ \\
\hline$K$ & energy density of cosmic rays in the galaxy in units of $\mathrm{erg}^{-1} \mathrm{~cm}^{-3}$ & $\$ 4.2$ \\
\hline$K_{0}$ & ratio of number densities of protons to electrons in cosmic rays accelerated in SNRs & $\S 4.2$ \\
\hline$d l$ & differential path length along the line of sight in units of pc & $\S 1.3$ \\
\hline$l$ & path length along the line of sight in units of pc & $\S 1.3$ \\
\hline & total path length along the line of sight & $\S 3.1$ \\
\hline$\left\langle L_{\mathrm{SMC}}\right\rangle$ & average depth of the SMC in pc & $\$ 3.3 .2$ \\
\hline$L_{\text {source }}$ & path length through the SMC to an extragalactic source in pc & $\$ 3.3 .2$ \\
\hline$L_{\text {synchrotron }}$ & depth of the synchrotron emitting layer in the SMC in $\mathrm{cm}$ & $\$ 4.2$ \\
\hline & average path length through the SMC to extragalactic sources in units of pc & $\$ 3.3 .2$ \\
\hline$\Delta L$ & standard deviation of $L_{\text {source }}$ through different sight lines in the SMC & $\$ 3.3 .2$ \\
\hline$l_{o}$ & Turbulence outer scale/ typical cell size in the SMC in units of pc & $\S 4.3, \S 6.2$ \\
\hline$\lambda$ & wavelength in meters & $\S 1.3$ \\
\hline
\end{tabular}


TABLE $1-$ Continued

\begin{tabular}{|c|c|c|}
\hline Symbol & Physical Quantity & Section in which first used \\
\hline$m$ & dynamo mode & $\S 6.4$ \\
\hline$m_{H}$ & mass of hydrogen atom & $\S 4.1$ \\
\hline$\xi\left(\lambda_{\mathrm{H} \alpha}\right)$ & extinction of the Milky Way and of the SMC at the wavelength of $\mathrm{H} \alpha$ & $\S 3.2$ \\
\hline$\nu$ & frequency in $\mathrm{Hz}$ & $\S 1.2$ \\
\hline$\hat{n}$ & unit vector normal to the SMC's HI disk & $\S 5$ \\
\hline $\mathrm{n}$ & number of sight lines through the SMC & $\$ 3.3 .4$ \\
\hline$n_{e}(l)$ & electron density distribution as a function of path length $l$ in units of $\mathrm{cm}^{-3}$ & $\S 1.3$ \\
\hline$\frac{c}{n_{e}}$ & average electron density along the line of sight & $\S 3.1$ \\
\hline$\left\langle n_{e}\right\rangle$ & mean electron density in the SMC in units of $\mathrm{cm}^{-3}$ & $\S 3.1$ \\
\hline$\overline{n_{e}^{2}}$ & average of the square of the electron density along the line of sight & $\S 3.2$ \\
\hline$n_{\text {cloud }}$ & electron density in an ionized gas cloud & $\$ 3.3 .2$ \\
\hline$\frac{c \text { cloud }}{n_{\text {clon }}}$ & average electron density in ionized gas clouds along the line-of-sight & $\$ 3.3 .2$ \\
\hline$n_{e_{\mathrm{cell}, \mathrm{i}}}$ & electron density in cell $i$ along the line of sight & $\S \mathrm{B}$ \\
\hline$n_{H}$ & number density of hydrogen in the ISM of the SMC & $\S 4.1$ \\
\hline$N_{\mathrm{HI}}$ & neutral hydrogen column density in units of $\mathrm{cm}^{2}$ & $\S 3.2$ \\
\hline$N$ & number of cells along a line of sight & $\S \mathrm{B}$ \\
\hline$\langle N\rangle$ & average number of cells when looking through the SMC along the line of sight & $\S \mathrm{B}$ \\
\hline$P_{i}$ & weights $=1 / \sigma_{B, i}^{2}$ & $\S 3.3 .4$ \\
\hline $\bar{P}$ & weighted mean of the weights $P_{i}$ & $\S 3.3 .4$ \\
\hline Q & quality of fit of the least square fit of $\Delta \phi$ against $\lambda^{2}$ & $\S 2.1$ \\
\hline $\mathrm{RM}$ & rotation measure in units of $\mathrm{rad} \mathrm{m}^{-2}$ & $\S 1.3$ \\
\hline $\mathrm{RM}_{\text {observed }}$ & observed RM of an extragalactic source & $\S 1.3$ \\
\hline $\mathrm{RM}_{\mathrm{SMC}}$ & rotation measure through the SMC & $\S 1.3$ \\
\hline $\mathrm{RM}_{\text {Intrinsic }}$ & intrinsic rotation measure of an extragalactic source & $\S 1.3$ \\
\hline $\mathrm{RM}_{\mathrm{IGM}}$ & rotation measure originating from the intergalactic medium & $\S 1.3$ \\
\hline $\mathrm{RM}_{\text {MilkyWay }}$ & rotation measure due to the Milky Way foreground & $\S 1.3$ \\
\hline$\langle\mathrm{RM}\rangle$ & weighted mean of the RM of extragalactic sources through the SMC & $\S \mathrm{B}$ \\
\hline $\mathrm{RM}_{1-\text { cell }}^{\prime}$ & rotation measure through 1 cell & $\S \mathrm{B}$ \\
\hline $\mathrm{RM}_{N-\text { cells }}$ & rotation measure through $\mathrm{N}$ cells & $\S \mathrm{B}$ \\
\hline$R_{\alpha}$ & parameter that characterizes the $\alpha$-effect & $\S 6.2$ \\
\hline$R_{\omega}$ & parameter that characterizes the $\omega$-effect & $\S 6.2$ \\
\hline$\rho$ & mass density of the phase of the ISM containing dust in $\mathrm{g} \mathrm{cm}^{-3}$ & $\S 1.4$ \\
\hline$\sigma_{\mathrm{RM}}$ & weighted standard deviation of RMs & $\S 4.3$ \\
\hline$\sigma_{\text {spatial,1D }}$ & one dimensional spatial dispersion of the radio pulsars in the SMC & $\S 3.1$ \\
\hline$\sigma_{v_{l o s}}^{2}$ & line of sight velocity dispersion of the phase of the ISM containing dust & $\S 1.4$ \\
\hline$S E M_{w}$ & weighted standard error in the mean line-of-sight magnetic field & $\$ 3.3 .4$ \\
\hline$s$ & radial distance from the center of the galaxy & $\S 6.2$ \\
\hline$T_{e}$ & electron temperature of the WIM in Kelvins & $\S 3.2$ \\
\hline$\tau_{\mathrm{H} \alpha, \mathrm{SMC}}$ & $\mathrm{H} \alpha$ optical depth of the SMC & $\S \mathrm{A}$ \\
\hline$\tau_{\mathrm{H} \alpha, \mathrm{MW}}$ & $\mathrm{H} \alpha$ optical depth of the Milky Way & $\S \mathrm{A}$ \\
\hline$\tau_{\mathrm{B}}$ & extinction optical depth in the optical B band & $\S 3.2$ \\
\hline$\tau_{\mathrm{H} \alpha}$ & optical depth of the SMC/Milky Way in the $\mathrm{H} \alpha$ line & $\S 3.2$ \\
\hline$\theta_{p}$ & position angle of starlight polarization in radians & $\S 1.4$ \\
\hline$\left\langle\theta_{p}\right\rangle$ & weighted mean of $\theta_{p}$ towards stars in the SMC & $\S 1.4$ \\
\hline$u_{0}$ & turbulent velocity in the galaxy in $\mathrm{cm} \mathrm{s}^{-1}$ & $\S 6.2$ \\
\hline$\Omega$ & angular velocity of galactic rotation & $\S 6.2$ \\
\hline
\end{tabular}


TABLE 2

PROPERTIES OF THE SMC

\begin{tabular}{|c|c|}
\hline Parameter & \\
\hline Right ascension $(\mathrm{J} 2000)^{a}$ & $01^{h} 05^{m} \mathrm{~b}$ \\
\hline Declination $(\mathrm{J} 2000)^{\text {a }}$ & $-72^{d} 49.7^{m} \mathrm{~b}$ \\
\hline Galactic coordinates $(1, b)$ & $(302.8,-44.6)^{\mathrm{c}}$ \\
\hline Galacto-centric coordinate $(\mathrm{X}, \mathrm{Y}, \mathrm{Z})(\mathrm{kpc})$ & $(15.3,-36.9,-43.3)^{c}$ \\
\hline Galacto-centric Space Velocity $\left(v_{X}, v_{Y}, v_{Z}\right)\left(\mathrm{km} \mathrm{s}^{-1}\right)$ & $(-87 \pm 48,-247 \pm 42,149 \pm 37)^{\mathrm{c}}$ \\
\hline Diameter on Sky & $\approx 7^{\circ \mathrm{d}}$ \\
\hline Distance $(\mathrm{kpc})$ & $\approx 60$ \\
\hline Inclination of $\mathrm{HI}$ disk & $40^{\circ} \pm 20^{\circ} \mathrm{b}$ \\
\hline Kinematic PA of major axis ${ }^{e}$ & $\sim 40^{\circ} \mathrm{b}$ \\
\hline HI Mass & $4.2 \times 10^{8} \mathrm{M}_{\odot}{ }^{\mathrm{b}}$ \\
\hline
\end{tabular}

\footnotetext{
a The apparent kinematic center of the SMC

${ }^{\mathrm{b}}$ Stanimirović et al. (2004)

${ }^{c}$ Kallivayalil et al. (2006)

d Westerlund (1990)

e The position angle (PA) is measured from north through east
} 
TABLE 3

Rotation Measures of Extragalactic Sources in the Observed Field

\begin{tabular}{|c|c|c|c|}
\hline Source Name ${ }^{a}$ & $\mathrm{RA}(\mathrm{J} 2000)(\mathrm{hms})$ & $\operatorname{DEC}(\mathrm{J} 2000)(\mathrm{dms})$ & $\mathrm{RM}\left(\mathrm{rad} \mathrm{m}^{-2}\right)$ \\
\hline 005 & $1: 41: 55.85$ & $-69: 41: 39.94$ & $-12 \pm 18$ \\
\hline 006 & $1: 41: 28.51$ & $-70: 16: 35.80$ & $+2 \pm 24$ \\
\hline 007 & $1: 41: 16.35$ & $-70: 15: 04.00$ & $+40 \pm 25$ \\
\hline 008 & $1: 33: 48.46$ & $-69: 28: 43.82$ & $+3 \pm 14$ \\
\hline 009 & $1: 33: 47.71$ & $-69: 28: 38.37$ & $+2 \pm 14$ \\
\hline 010 & $1: 33: 39.56$ & $-69: 28: 37.32$ & $-16 \pm 20$ \\
\hline 011 & $1: 28: 45.87$ & $-69: 36: 16.12$ & $-5 \pm 15$ \\
\hline 012 & $1: 29: 44.77$ & $-70: 35: 31.04$ & $+18 \pm 24$ \\
\hline 013 & $1: 21: 49.98$ & $-69: 56: 43.37$ & $+21 \pm 9$ \\
\hline 014 & $1: 21: 44.40$ & $-69: 57: 21.11$ & $+28 \pm 19$ \\
\hline 015 & $1: 28: 16.99$ & $-75: 12: 58.75$ & $+35 \pm 13$ \\
\hline 016 & $1: 28: 08.78$ & $-75: 12: 51.83$ & $+42 \pm 14$ \\
\hline 017 & $1: 22: 57.30$ & $-75: 15: 04.66$ & $+30 \pm 11$ \\
\hline $018^{*}$ & $1: 10: 35.37$ & $-72: 28: 07.70$ & $-82 \pm 27$ \\
\hline $019^{*}$ & $1: 10: 55.99$ & $-73: 14: 11.49$ & $-37 \pm 14$ \\
\hline $020^{*}$ & $1: 10: 50.67$ & $-73: 14: 25.42$ & $+3 \pm 13$ \\
\hline $021^{*}$ & 1:10:48.65 & $-73: 14: 29.14$ & $+6 \pm 5$ \\
\hline 022 & $1: 01: 32.49$ & $-69: 39: 14.21$ & $-8 \pm 32$ \\
\hline 023 & 1:03:33.17 & $-75: 06: 57.46$ & $+6 \pm 33$ \\
\hline 024 & $0: 57: 15.86$ & $-70: 40: 47.01$ & $+44 \pm 24$ \\
\hline $025^{*}$ & $0: 56: 45.05$ & $-72: 51: 59.06$ & $-30 \pm 41$ \\
\hline 026 & $0: 52: 23.71$ & $-75: 25: 48.72$ & $-5 \pm 17$ \\
\hline 027 & $0: 47: 40.77$ & $-75: 30: 11.36$ & $+21 \pm 4$ \\
\hline 028 & $0: 42: 38.74$ & $-70: 01: 34.56$ & $+34 \pm 24$ \\
\hline 029 & $0: 40: 47.68$ & $-71: 45: 59.63$ & $+18 \pm 26$ \\
\hline 030 & $0: 39: 39.59$ & $-71: 41: 42.44$ & $+41 \pm 21$ \\
\hline 031 & $0: 38: 01.40$ & $-72: 52: 10.65$ & $+21 \pm 24$ \\
\hline 032 & $0: 37: 54.79$ & $-72: 51: 56.66$ & $+1 \pm 13$ \\
\hline 033 & $0: 34: 28.44$ & $-73: 35: 26.77$ & $+12 \pm 24$ \\
\hline 034 & $0: 34: 25.83$ & $-73: 35: 13.86$ & $+10 \pm 27$ \\
\hline 035 & $0: 34: 14.98$ & $-73: 33: 28.08$ & $+43 \pm 18$ \\
\hline 036 & $0: 34: 15.58$ & $-73: 33: 34.29$ & $+53 \pm 14$ \\
\hline 037 & $0: 34: 15.20$ & $-73: 33: 16.16$ & $+42 \pm 22$ \\
\hline 038 & $0: 29: 20.10$ & $-75: 40: 08.70$ & $+7 \pm 48$ \\
\hline 039 & $0: 32: 31.35$ & $-73: 06: 50.38$ & $+50 \pm 30$ \\
\hline 040 & $0: 32: 30.92$ & $-69: 24: 28.54$ & $+9 \pm 48$ \\
\hline 041 & $0: 26: 08.35$ & $-73: 23: 15.03$ & $+51 \pm 20$ \\
\hline 042 & $0: 24: 11.37$ & $-73: 57: 15.95$ & $+54 \pm 8$ \\
\hline 043 & $0: 23: 37.31$ & $-73: 55: 30.19$ & $+51 \pm 8$ \\
\hline 044 & $0: 22: 21.97$ & $-74: 28: 21.27$ & $+40 \pm 9$ \\
\hline 045 & $0: 29: 28.04$ & $-69: 34: 35.04$ & $+24 \pm 12$ \\
\hline 046 & $0: 29: 26.11$ & $-69: 34: 46.23$ & $+26 \pm 17$ \\
\hline 047 & $0: 22: 15.40$ & $-74: 28: 14.62$ & $+63 \pm 19$ \\
\hline 048 & $0: 22: 02.76$ & $-74: 27: 22.53$ & $+40 \pm 23$ \\
\hline 082 & $0: 14: 24.12$ & $-73: 39: 05.15$ & $+29 \pm 33$ \\
\hline 087 & $0: 11: 56.37$ & $-73: 49: 56.30$ & $+84 \pm 41$ \\
\hline 115 & $0: 31: 36.77$ & $-70: 33: 18.49$ & $+67 \pm 70$ \\
\hline 116 & $0: 28: 41.87$ & $-70: 45: 19.61$ & $+60 \pm 38$ \\
\hline 117 & $1: 39: 48.32$ & $-69: 33: 27.44$ & $+16 \pm 43$ \\
\hline 118 & 1:18:09.61 & $-69: 46: 04.36$ & $+22 \pm 51$ \\
\hline 119 & $1: 18: 16.62$ & $-69: 51: 50.35$ & $+20 \pm 52$ \\
\hline 120 & $1: 22: 45.57$ & $-69: 44: 18.52$ & $+31 \pm 38$ \\
\hline $121^{*}$ & $1: 10: 45.44$ & $-72: 28: 52.19$ & $-69 \pm 44$ \\
\hline 122 & 1:19:17.56 & $-71: 05: 42.40$ & $+43 \pm 52$ \\
\hline 123 & $0: 34: 01.18$ & $-70: 26: 29.06$ & $+41 \pm 46$ \\
\hline 124 & $0: 21: 38.21$ & $-69: 26: 16.61$ & $+12 \pm 47$ \\
\hline 125 & $0: 28: 36.06$ & $-69: 33: 40.34$ & $+16 \pm 34$ \\
\hline 126 & $0: 46: 39.63$ & $-69: 57: 12.48$ & $+20 \pm 32$ \\
\hline 127 & $0: 42: 24.66$ & $-70: 02: 47.47$ & $-5 \pm 30$ \\
\hline $128^{*}$ & $0: 49: 34.99$ & $-72: 19: 03.34$ & $-204 \pm 76$ \\
\hline 130 & $0: 26: 06.34$ & $-73: 23: 10.97$ & $+46 \pm 27$ \\
\hline 131 & $1: 37: 02.32$ & $-73: 04: 17.75$ & $-16 \pm 32$ \\
\hline 132 & $1: 37: 05.69$ & $-73: 04: 14.74$ & $+4 \pm 51$ \\
\hline 133 & $1: 46: 39.13$ & $-72: 48: 55.80$ & $-8 \pm 49$ \\
\hline $134^{*}$ & $1: 29: 30.54$ & $-73: 33: 12.41$ & $-385 \pm 56$ \\
\hline $135^{*}$ & $1: 33: 30.53$ & $-73: 03: 06.52$ & $-101 \pm 45$ \\
\hline $136^{*}$ & $0: 56: 43.08$ & $-72: 52: 17.22$ & $+23 \pm 48$ \\
\hline 137 & $0: 26: 05.65$ & $-73: 23: 10.61$ & $+38 \pm 28$ \\
\hline 138 & $0: 34: 10.03$ & $-70: 25: 19.64$ & $-15 \pm 47$ \\
\hline 139 & $0: 24: 28.98$ & $-70: 09: 29.40$ & $+41 \pm 22$ \\
\hline
\end{tabular}

a Missing source names are polarized sources identified by the SFIND source finding algorithm which do not correspond to real sources in Stokes $I$ image.

* The source is behind the SMC. 
TABLE 4

Radio Pulsars in the SMALl Magellanic Cloud

\begin{tabular}{|c|c|c|c|c|c|c|}
\hline Name & RA (J2000) a & $\operatorname{DEC}(\mathrm{J} 2000)^{a}$ & $\mathrm{DM}\left(\mathrm{pc} \mathrm{cm^{-3 } ) ^ { \mathrm { a } }}\right.$ & $\mathrm{DM}_{\text {foreground }}\left(\mathrm{pc} \mathrm{cm}^{-3}\right)^{\mathrm{b}}$ & $\mathrm{DM}_{\mathrm{SMC} \text { pulsar }}\left(\mathrm{pc} \mathrm{cm}^{-3}\right)^{\mathrm{c}}$ & $\mathrm{RM}\left(\mathrm{rad} \mathrm{m}^{-2}\right)^{\mathrm{a}}$ \\
\hline PSR J0045-7042 & $00: 45: 25.69$ & $-70: 45: 07.1$ & $70 \pm 3$ & 40.75 & 29 & \\
\hline PSR J0045-7319 & 00:45:33.16 & $-73: 19: 03.0$ & $105.4 \pm 0.7$ & 41.21 & 64.2 & $-14 \pm 27$ \\
\hline PSR J0111-7131 & 01:11:28.77 & $-71: 31: 46.8$ & $76 \pm 3$ & 42.65 & 33 & \\
\hline PSR J0113-7200 & 01:13:11.09 & $-72: 20: 32.2$ & $125.49 \pm 0.03$ & 42.92 & 85.57 & $\ldots$ \\
\hline PSR J0131-7310 & 01:31:28.51 & $-73: 10: 09.0$ & $205.2 \pm 0.7$ & 41.94 & 163.3 & $\ldots$ \\
\hline
\end{tabular}

${ }^{a}$ Manchester et al. $(2005,2006)$

b NE 2001 Galactic Free Electron Model (Cordes \& Lazio 2002)

c Dispersion measure of pulsars after the removal of Galactic foreground contribution 
Mao et al.

TABLE 5

ROTATION MEASURES OF SOURCES BEHIND THE SMC AFTER FOREGROUND RM SUBTRACTION

\begin{tabular}{lr}
\hline \hline Source Name & \multicolumn{1}{r}{$\mathrm{RM}\left(\mathrm{rad} \mathrm{m}^{-2}\right)$} \\
\hline 018 & $-100 \pm 30$ \\
019 & $-60 \pm 10$ \\
020 & $-20 \pm 10$ \\
021 & $-15 \pm 6$ \\
025 & $-60 \pm 40$ \\
121 & $-90 \pm 40$ \\
128 & $-230 \pm 80$ \\
134 & $-400 \pm 60$ \\
135 & $-110 \pm 50$ \\
136 & $0 \pm 50$ \\
& \\
\hline
\end{tabular}

TABLE 6

Foreground CORRECTED STARLIGHT POLARIZATION DATA

\begin{tabular}{|c|c|c|c|c|}
\hline Star ID & $\mathrm{RA}(\mathrm{hm})^{\mathrm{b}}$ & $\mathrm{DEC}(\mathrm{dm})^{\mathrm{b}}$ & Position Angle (Degrees) ${ }^{\mathrm{c}}$ & Used in the C-F method? \\
\hline 3 & $0: 42.3$ & $-73: 32$ & $-6 \pm 90$ & yes \\
\hline 7 & $0: 44.9$ & $-73: 48$ & $41 \pm 34$ & yes \\
\hline 12 & $0: 46.3$ & $-72: 58$ & $33 \pm 25$ & yes \\
\hline 18 & $0: 47.0$ & $-73: 17$ & $48 \pm 14$ & yes \\
\hline 19 & $0: 47.0$ & $-73: 23$ & $74 \pm 13$ & yes \\
\hline 23 & $0: 47.5$ & $-73: 12$ & $40 \pm 42$ & yes \\
\hline 26 & $0: 48.0$ & $-72: 9$ & $9 \pm 29$ & yes \\
\hline 27 & $0: 48.2$ & $-73: 30$ & $39 \pm 62$ & yes \\
\hline 31 & $0: 49.0$ & $-73: 4$ & $-10 \pm 15$ & yes \\
\hline 33 & $0: 49.3$ & $-73: 16$ & $-81 \pm 6$ & yes \\
\hline 35 & $0: 49.4$ & $-72: 46$ & $18 \pm 13$ & yes \\
\hline 39 & $0: 49.6$ & $-73: 37$ & $-32 \pm 31$ & yes \\
\hline 40 & $0: 49.7$ & $-73: 36$ & $17 \pm 11$ & yes \\
\hline 45 & $0: 50.5$ & $-72: 31$ & $30 \pm 9$ & yes \\
\hline 46 & $0: 50.5$ & $-72: 15$ & $19 \pm 17$ & yes \\
\hline Anon(1) & $0: 51.4$ & $-73: 24$ & $83 \pm 10$ & yes \\
\hline 54 & $0: 52.0$ & $-73: 15$ & $-4 \pm 25$ & yes \\
\hline 56 & $0: 52.0$ & $-72: 46$ & $-12 \pm 19$ & yes \\
\hline 55 & $0: 52.2$ & $-71: 47$ & $-11 \pm 55$ & yes \\
\hline Anon(2) & $0: 52.3$ & $-73: 21$ & $63 \pm 16$ & yes \\
\hline 59 & $0: 54.0$ & $-72: 17$ & $18 \pm 42$ & yes \\
\hline 62 & $0: 55.3$ & $-73: 3$ & $38 \pm 14$ & yes \\
\hline 68 & $0: 56.7$ & $-71: 28$ & $-61 \pm 20$ & yes \\
\hline 67 & $0: 56.7$ & $-72: 24$ & $40 \pm 41$ & yes \\
\hline 74 & $0: 57.9$ & $-72: 34$ & $17 \pm 5$ & yes \\
\hline 78 & $0: 58.6$ & $-72: 18$ & $15 \pm 27$ & yes \\
\hline 80 & $0: 58.6$ & $-72: 19$ & $-19 \pm 23$ & yes \\
\hline 82 & $0: 59.0$ & $-72: 53$ & $10 \pm 124$ & yes \\
\hline 85 & $0: 59.3$ & $-72: 22$ & $-35 \pm 26$ & yes \\
\hline 89 & $0: 59.9$ & $-71: 41$ & $18 \pm 149$ & yes \\
\hline 97 & $1: 0.5$ & $-72: 41$ & $83 \pm 34$ & yes \\
\hline 98 & $1: 0.5$ & $-72: 25$ & $25 \pm 92$ & yes \\
\hline 103 & $1: 1.2$ & $-72: 44$ & $20 \pm 47$ & yes \\
\hline 105 & $1: 1.9$ & $-72: 15$ & $-21 \pm 26$ & yes \\
\hline 106 & $1: 2.0$ & $-72: 18$ & $-26 \pm 28$ & yes \\
\hline 107 & $1: 2.1$ & $-71: 56$ & $-20 \pm 24$ & yes \\
\hline 108 & $1: 2.6$ & $-72: 14$ & $-67 \pm 26$ & no \\
\hline 114 & $1: 4.0$ & $-72: 14$ & $-66 \pm 131$ & no \\
\hline 117 & $1: 4.1$ & $-72: 16$ & $20 \pm 30$ & yes \\
\hline 118 & $1: 4.2$ & $-72: 10$ & $2 \pm 13$ & yes \\
\hline 119 & $1: 4.2$ & $-72: 48$ & $33 \pm 22$ & yes \\
\hline 120 & $1: 4.5$ & $-73: 11$ & $61 \pm 36$ & no \\
\hline 121 & $1: 4.6$ & $-72: 56$ & $-50 \pm 44$ & yes \\
\hline 124 & $1: 5.2$ & $-72: 27$ & $-57 \pm 19$ & yes \\
\hline
\end{tabular}


TABLE 6 - Continued

\begin{tabular}{lllcc}
\hline \hline Star ID $^{\mathrm{a}}$ & RA(hm $^{\mathrm{b}}$ & DEC(dm) & Position Angle (Degrees) $^{\mathrm{c}}$ & Used in the C-F method? $^{\mathrm{b}}$ \\
\hline 128 & $1: 5.7$ & $-72: 30$ & $-37 \pm 46$ & yes \\
130 & $1: 6.5$ & $-72: 36$ & $-62 \pm 12$ & yes \\
136 & $1: 8.2$ & $-73: 11$ & $-7 \pm 22$ & yes \\
137 & $1: 8.3$ & $-72: 40$ & $-19 \pm 20$ & yes \\
138 & $1: 8.5$ & $-72: 33$ & $-86 \pm 52$ & no \\
145 & $1: 10.7$ & $-72: 39$ & $20 \pm 88$ & yes \\
146 & $1: 11.0$ & $-72: 15$ & $-47 \pm 28$ & yes \\
149 & $1: 1.8$ & $-71: 17$ & $-6 \pm 10$ & no \\
150 & $1: 12.3$ & $-72: 54$ & $-12 \pm 17$ & yes \\
152 & $1: 12.8$ & $-73: 28$ & $-72 \pm 21$ & yes \\
154 & $1: 13.6$ & $-73: 21$ & $21 \pm 13$ & yes \\
155 & $1: 14.2$ & $-73: 28$ & $14 \pm 11$ & yes \\
156 & $1: 14.8$ & $-73: 28$ & $84 \pm 44$ & no \\
157 & $1: 15.2$ & $-73: 29$ & $40 \pm 26$ & yes \\
159 & $1: 15.3$ & $-73: 29$ & $51 \pm 55$ & yes \\
163 & $1: 19.2$ & $-72: 48$ & $-13 \pm 32$ & yes \\
165 & $1: 19.8$ & $-73: 14$ & $44 \pm 98$ & yes \\
168 & $1: 21.1$ & $-72: 54$ & $77 \pm 18$ & yes \\
166 & $1: 21.2$ & $-74: 6$ & $-17 \pm 26$ & yes \\
181 & $1: 28.7$ & $-72: 50$ & $20 \pm 13$ & yes \\
187 & $1: 30.3$ & $-73: 31$ & $-1 \pm 23$ & yes \\
189 & $1: 30.7$ & $-72: 56$ & $46 \pm 36$ & no \\
191 & $1: 41.2$ & $-73: 58$ & $-19 \pm 26$ & no \\
193 & $1: 43.8$ & $-74: 47$ & $32 \pm 7$ & no \\
194 & $1: 44.7$ & $-74: 39$ & $8 \pm 28$ & no \\
196 & $1: 48.8$ & $-74: 8$ & $-2 \pm 21$ & no \\
198 & $1: 50.8$ & $-74: 3$ & $69 \pm 11$ & no \\
202 & $1: 52.8$ & $-74: 2$ & $50 \pm 29$ & no \\
205 & $1: 53.2$ & $-74: 10$ & $18 \pm 120$ & no \\
215 & $2: 13.3$ & $-74: 38$ & $-19 \pm 15$ & yes \\
HV 821 & $0: 40.9$ & $-73: 52$ & $36 \pm 23$ & \\
NGC 330 & $0: 55.5$ & $-72: 37$ & $25 \pm 15$ & \\
& & & & \\
\hline & & & & \\
\hline
\end{tabular}

a The star IDs have been taken from the catalogues of Sanduleak \& Philip (1968); Sanduleak $(1968,1969)$ whenever available. b The positions of stars are in epoch 1975.

${ }^{c}$ The position angle is measured counterclockwise with respect to the great circle joining the SMC to the LMC. 
Mao et al.

TABLE 7

MEASUREMENT OF THE LINE OF SIGHT MAGNETIC FIELD STRENGTH THROUGH THE SMC

\begin{tabular}{lccc}
\hline \hline Source Name & Model $1 \overline{B_{\|}}(\mu \mathrm{G})$ & Model $2 \overline{B_{\|}}(\mu \mathrm{G})$ & Model $3 \overline{B_{\|}}(\mu \mathrm{G})$ \\
\hline 018 & $-0.8 \pm 0.2$ & $-0.2 \pm 0.1$ & $-0.4 \pm 0.1$ \\
019 & $-0.4 \pm 0.1$ & $-0.4 \pm 0.1$ & $-0.4 \pm 0.1$ \\
020 & $-0.1 \pm 0.1$ & $-0.1 \pm 0.1$ & $-0.1 \pm 0.1$ \\
021 & $-0.10 \pm 0.05$ & $-0.10 \pm 0.04$ & $-0.10 \pm 0.04$ \\
025 & $-0.4 \pm 0.3$ & $-0.1 \pm 0.1$ & $-0.2 \pm 0.2$ \\
121 & $-0.7 \pm 0.3$ & $-0.2 \pm 0.1$ & $-0.4 \pm 0.2$ \\
128 & $-1.8 \pm 0.6$ & $-0.5 \pm 0.2$ & $-1.0 \pm 0.3$ \\
134 & $-3.0 \pm 0.4$ & $-0.09 \pm 0.01$ & $-0.5 \pm 0.1$ \\
135 & $-0.9 \pm 0.3$ & $-2.7 \pm 1.1$ & $-1.5 \pm 0.6$ \\
136 & $0.0 \pm 0.4$ & $0.0 \pm 0.1$ & $0.0 \pm 0.2$ \\
\hline
\end{tabular}




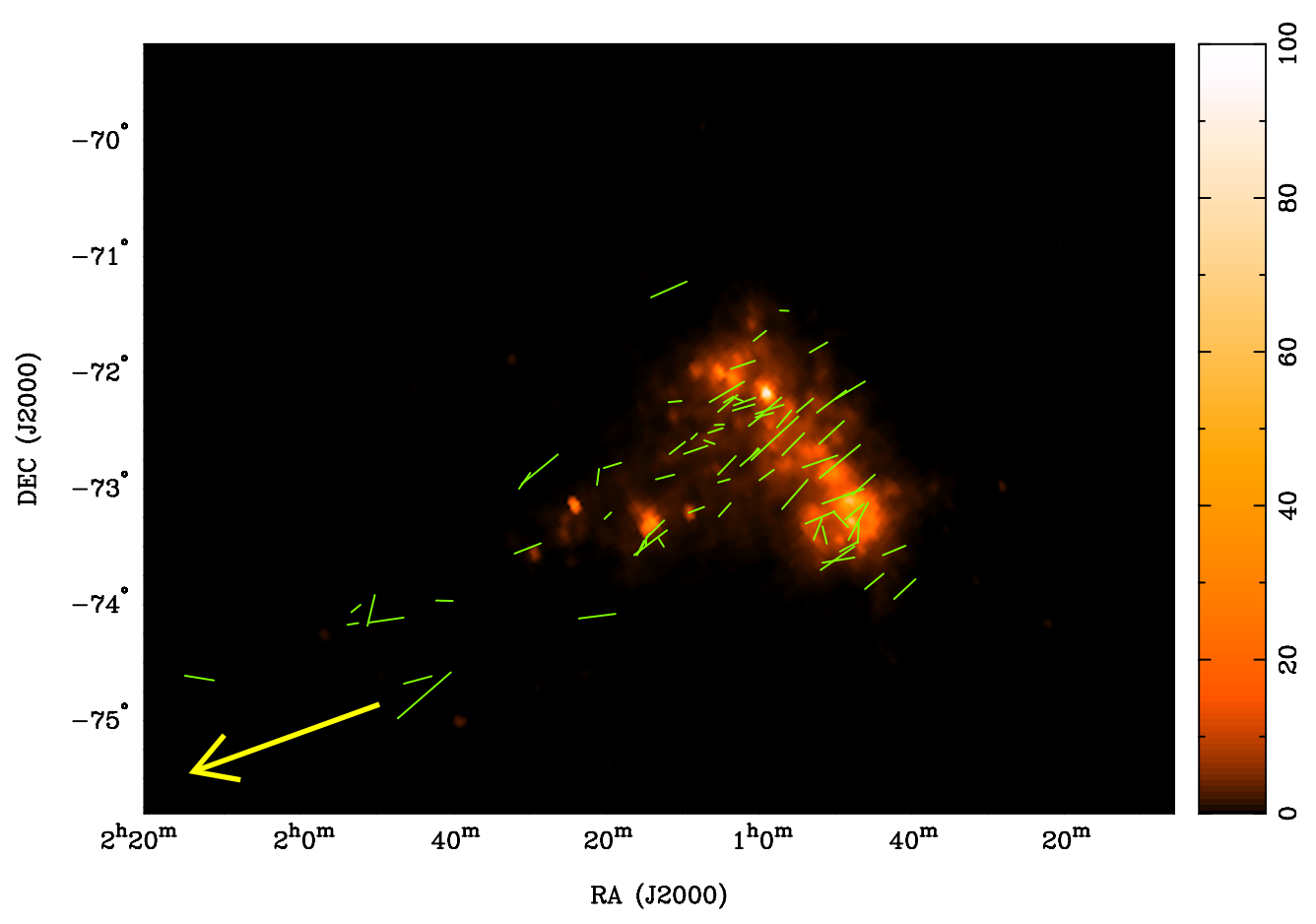

FIG. 1.- Optical starlight polarization "vectors", before foreground correction, towards stars in the SMC and in part of the Magellanic Bridge (Mathewson \& Ford 1970a), overlaid on an IRAS $60 \mu \mathrm{m}$ image. The color scale to the right of the image is the IR flux in units of MJy per steradian. The orientation of the line segments indicates the polarization position angle whereas the length of the line segments is proportional to the polarized fraction. The eastmost line segment corresponds to a star with observed polarized fraction of $0.51 \%$. Each star is located at the center of its corresponding line segment. Stars are indicated at their positions as of epoch 1975, as given in Mathewson \& Ford (1970a). The yellow arrow indicates the direction towards the LMC. 

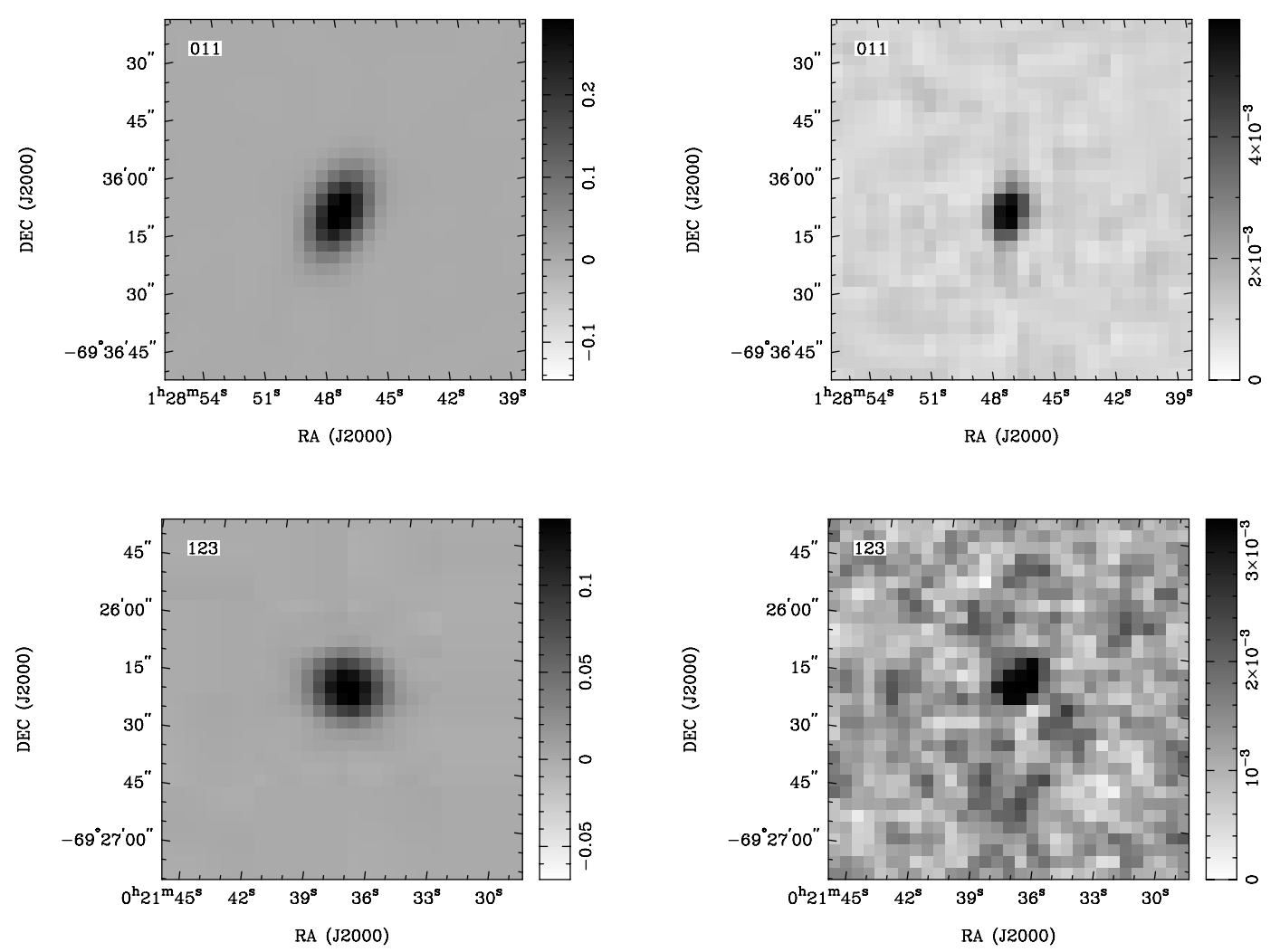

FIG. 2.- Two examples of polarized extragalactic background sources detected in our survey, source 011 and source 123 in Table 3 . The left panel shows the total intensity, while the right panel shows the linear polarized intensity. Grey scale in units of Jy is shown to the left of each figure. 

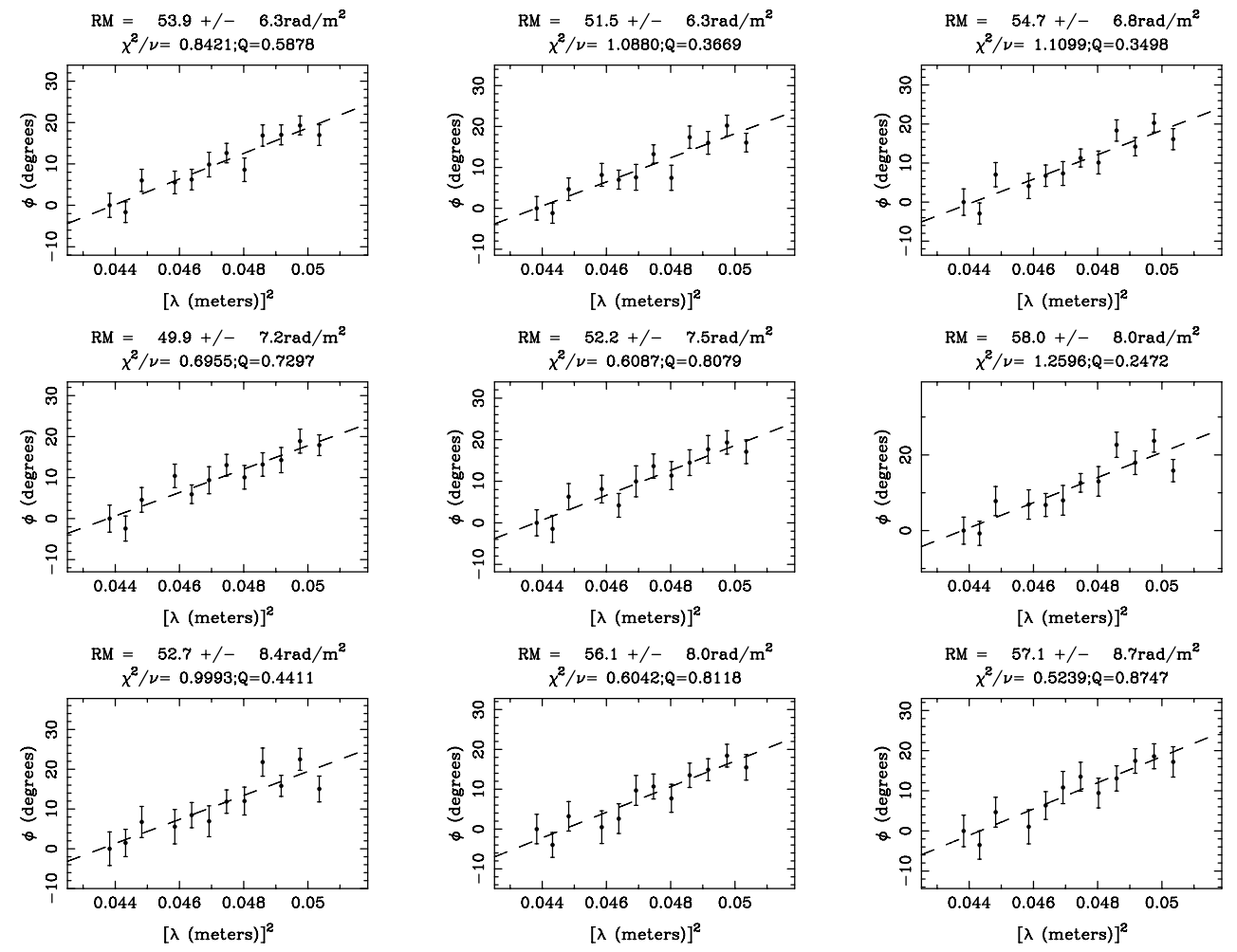

FIG. 3. - Least square fits of polarization angle against wavelength squared for the nine brightest pixels in polarization for extragalactic source 042 in Table 3 . The plot at the upper left shows the fit to the brightest pixel, while the plot at the lower right corresponds to the fit of the faintest pixel. The slope of the least square fit gives the value of the RM, which is indicated above each plot. The reduced $\chi^{2}$ and the quality of fit (Q) for each pixel are also displayed. 


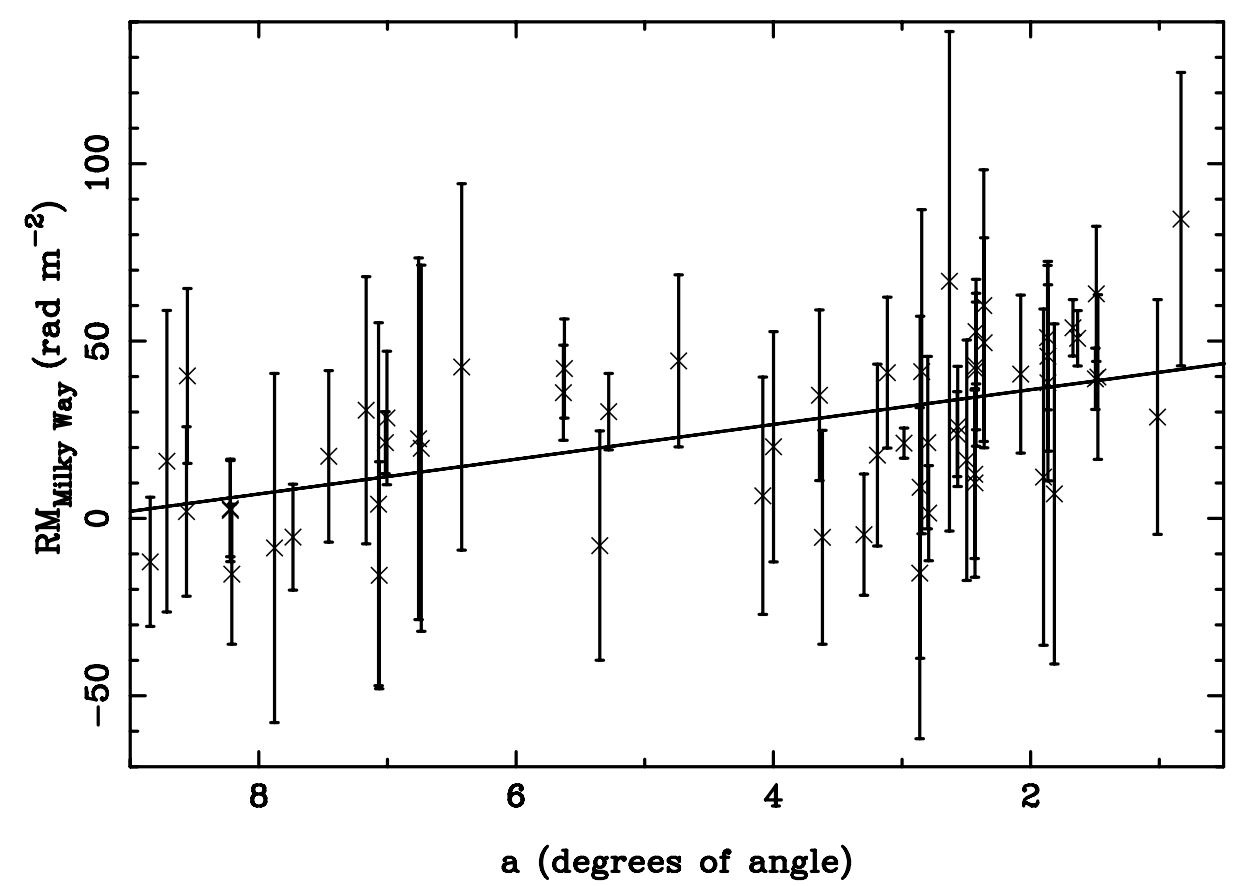

FIG. 4.- Foreground RM fit to the 60 extragalactic sources in Table 3 whose projections on the sky lie outside the SMC. The foreground RM can be least square fitted as a linear function of right ascension. The best fit is $\mathrm{RM}_{\text {MilkyWay }}=46.1-4.9 \times \mathrm{a} \mathrm{rad} \mathrm{m}{ }^{-2}$ with a reduced $\chi^{2}$ of 0.88 , where a is the offset eastward from right ascension of 0 in degrees. 


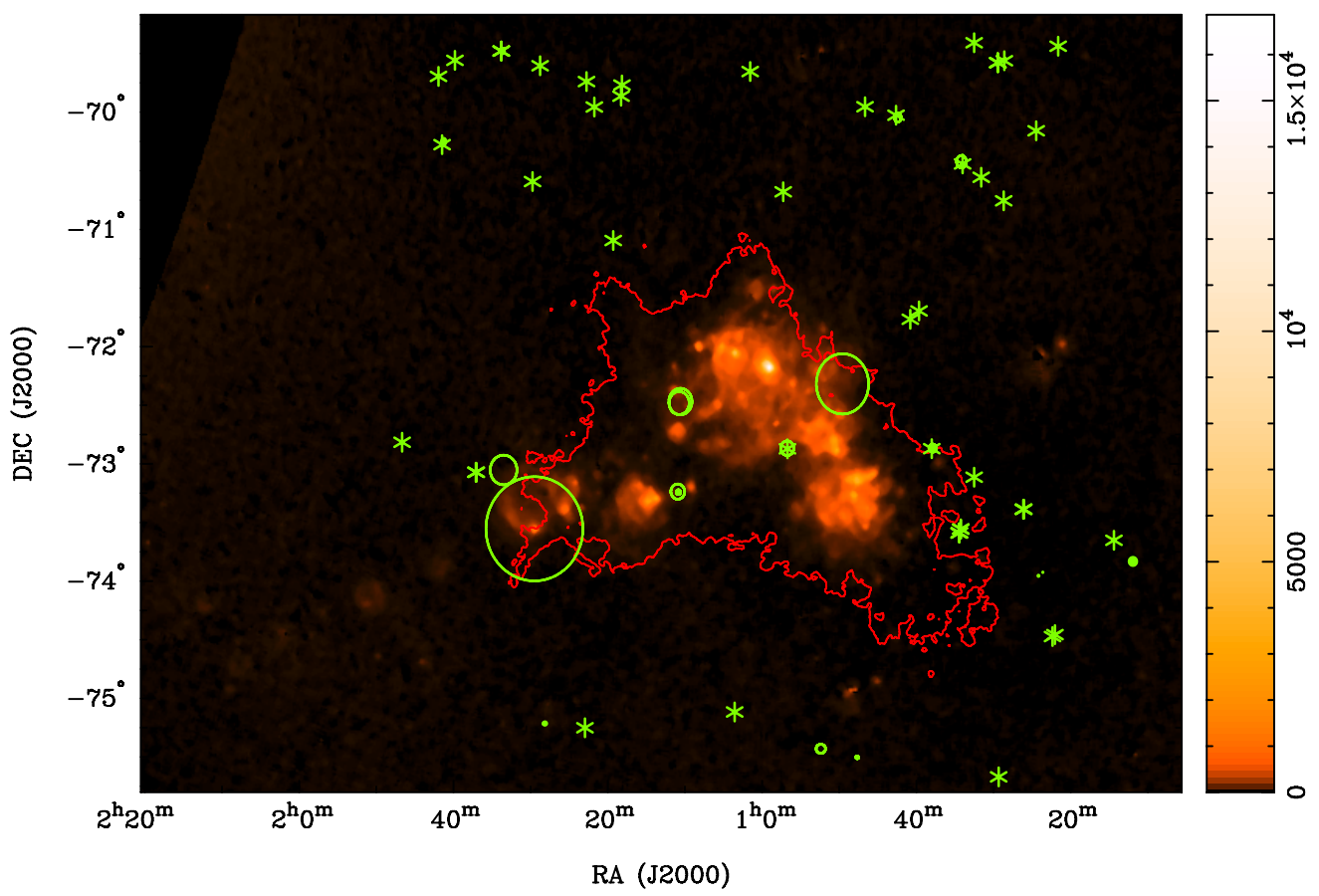

FIG. 5.- Distribution of foreground corrected RMs overlaid on smoothed and continuum subtracted $\mathrm{H} \alpha$ emission from the SHASSA survey (Gaustad et al. 2001). The color scale is in units of $\mathrm{dR}$. The contour represents an HI column density of $2 \times 10^{21}$ atoms cm ${ }^{-2}$ (Stanimirović et al. 2004). Closed and open circles represent positive and negative rotation measures respectively. Asterisks denote RMs that are consistent with zero within their uncertainties. The center of the symbol marks the position of the extragalactic source. The diameter of a circle is proportional to the value of $|\mathrm{RM}|$ at that position. The largest open circle in the above figure represents a RM of $-400 \mathrm{rad} \mathrm{m}^{-2}$. The consistent pattern of negative RMs projected against the SMC indicates that the SMC has a significant coherent magnetic field along the line of sight, directed away from us. 


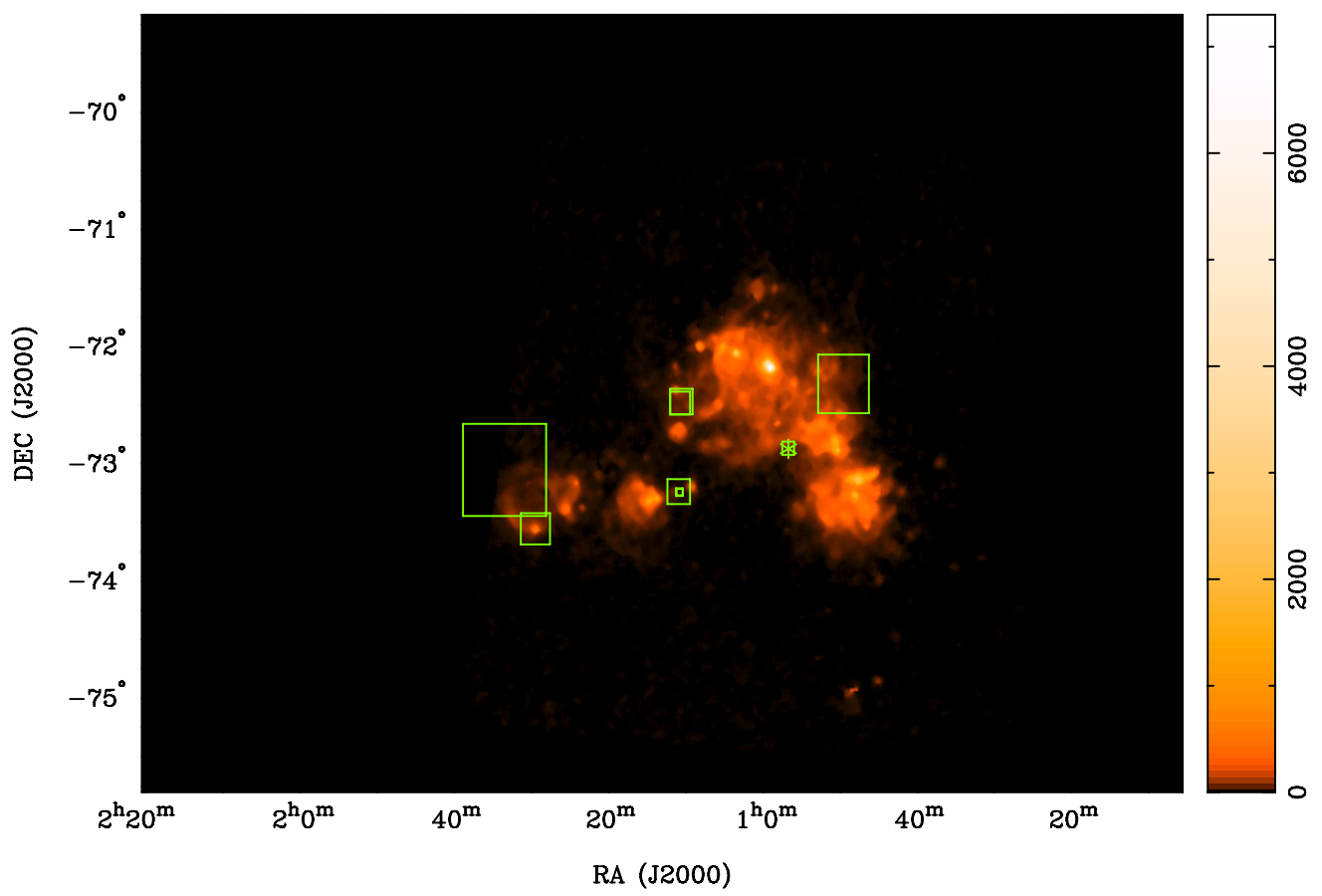

FIG. 6. - The line-of-sight magnetic field strength through the SMC derived using ionized gas model 3 ( $\S 3.3 .3$ ). The image is the extinction corrected emission measure map of the SMC, in units of $\mathrm{pc} \mathrm{cm}^{-3}$ (as shown by the scale bar to the right of the image), derived from the SHASSA H $\alpha$ survey. Open squares denote magnetic fields whose line of sight component direct away from us while the asterisk denotes a magnetic field strength consistent with zero within one standard deviation. The centre of the squares mark the positions of the extragalactic sources. The length of a side of the square is proportional to the line of sight magnetic field strength. The largest open square in the above figure represents a field strength of $-2.0 \mu \mathrm{G}$. This figure illustrates that the SMC hosts a large scale coherent magnetic field of the order of $\sim 0.2 \mu \mathrm{G}$. 